\title{
Maass relations for Saito-Kurokawa lifts of higher levels
}

\author{
Jolanta Marzec ${ }^{1}$
}

Received: 19 March 2019 / Accepted: 17 January 2020 / Published online: 22 July 2020

(c) The Author(s) 2020

\begin{abstract}
It is known that among Siegel modular forms of degree 2 and level 1 the only functions that violate the Ramanujan conjecture are Saito-Kurokawa lifts of modular forms of level 1 . These are precisely the functions whose Fourier coefficients satisfy Maass relations. More generally, the Ramanujan conjecture for $\mathrm{GSp}_{4}$ is predicted to fail only in case of CAP representations. It is not known though whether the associated Siegel modular forms (of various levels) still satisfy a version of Maass relations. We show that this is indeed the case for the ones related to P-CAP representations. Our method generalises an approach of Pitale, Saha and Schmidt who employed representationtheoretic techniques to (re)prove this statement in case of level 1. In particular, we compute and express certain values of a global Bessel period in terms of Fourier coefficients of the associated Siegel modular form. Moreover, we derive a local-global relation satisfied by Bessel periods, which allows us to combine those computations with a characterization of local components of CAP representations.
\end{abstract}

Keywords Bessel period - CAP representations - Maass relations - Saito-Kurokawa lifts $\cdot$ Siegel modular forms

Mathematics Subject Classification 11F30 1 11F46 1 11F70

\section{Introduction}

It is known that a Siegel modular form $F$ is a (classical) Saito-Kurokawa lift of an elliptic modular form $f$ if and only if its Fourier coefficients satisfy the Maass relations

Open Access funding provided by Projekt DEAL. The author's studies and research were possible thanks to a funding provided by the Engineering and Physical Sciences Research Council.

\footnotetext{
$凶$ Jolanta Marzec

marzec@mathematik.tu-darmstadt.de

1 Department of Mathematics, Technische Universität Darmstadt, Schlossgartenstraße 7, 64289

Darmstadt, Germany
} 


$$
a\left(F,\left(\begin{array}{cc}
a & b / 2 \\
b / 2 & c
\end{array}\right)\right)=\sum_{r \mid \operatorname{gcd}(a, b, c)} r^{k-1} a\left(F,\left(\begin{array}{cc}
\frac{a c}{r^{2}} & \frac{b}{2 r} \\
\frac{b}{2 r} & 1
\end{array}\right)\right) .
$$

The classical cuspidal Saito-Kurokawa lift of weight $k$ is a lift from a cuspidal modular form $f \in S_{2 k-2}^{(1)}\left(\mathrm{SL}_{2}(\mathbb{Z})\right)$ with $k$ even; it is a cuspidal Siegel modular form $F \in S_{k}^{(2)}\left(\operatorname{Sp}_{4}(\mathbb{Z})\right)$. The first construction of such a lift was given by Maass in [10] using correspondences between Siegel and classical modular forms, Jacobi forms and modular forms of half-integral weight (see also [5]). However, Saito-Kurokawa lifts can be also constructed using representation theory [12,21]. The advantage of the latter is that it can be easily generalised to lifts of modular forms of higher level, and also with an odd weight. In this case, if $k$ is even, ${ }^{1}$ for $f \in S_{2 k-2}^{(1)}\left(\Gamma_{0}(N)\right)$ with $N$ square-free there exists a cuspidal Siegel modular form of weight $k$ invariant under the action of a congruence subgroup of $\mathrm{GSp}_{4}(\mathbb{Z})$ such that its spinor $L$-function is given by

$$
L(s, F)=C(s) L(s, f) \zeta(s-k+1) \zeta(s-k+2),
$$

where $C(s)$ comes from a contribution at $p=\infty$ and $p \mid N$ (for a precise statement see [22, Theorem 5.2]. ${ }^{2}$ ) This does not tell us though anything about the coefficients of $F$ and whether they satisfy similar Maass relations. Pitale et al. showed in [17] that this is indeed the case if $F \in S_{k}^{(2)}\left(\operatorname{Sp}_{4}(\mathbb{Z})\right)$ is a Hecke eigenform.

From a representation theoretic point of view, a Saito-Kurokawa lift produces from a cuspidal automorphic representation $\pi$ of $\mathrm{PGL}_{2}(\mathbb{A})$ a cuspidal automorphic representation $\Pi$ of $\mathrm{PGSp}_{4}(\mathbb{A})$; we can think of $f$ and $F$ as vectors of matching weight in the vector spaces of $\pi$ and $\Pi$. What is important is that any representation $\Pi$ we obtain via this (generalised) Saito-Kurokawa lifting is a CAP representation.

More precisely, consider a cuspidal Siegel modular form $F$ of level $\Gamma_{0}\left(N_{1}, N_{2}\right)$ (see notation below). We say that $F$ is associated to a CAP representation if the following are true.

(1) The adelisation of $F$ gives rise to an irreducible automorophic representation $\Pi$ of $\mathrm{GSp}_{4}(\mathbb{A})$.

(2) The representation $\Pi$ is equivalent at almost all places to a constituent of a globally induced representation from a proper parabolic subgroup of $\mathrm{GSp}_{4}$.

Furthermore, we say that $F$ is associated to a P-CAP representation if the proper parabolic subgroup above is the Siegel parabolic subgroup. The classical SaitoKurokawa lifts correspond exactly to the P-CAP representations. It is known (see [15, Corollary 4.5]) that if $k \geq 3$, then $F \in S_{k}^{(2)}\left(\Gamma_{0}(1, N)\right)$ that is associated to a CAP representation is automatically associated to a P-CAP representation. If $k=1$ or 2 , one also has CAP representations associated to other parabolics (the so-called B-CAP and Q-CAP representations).

\footnotetext{
1 If $k$ is odd, the construction leads to a non-holomorphic function (cf. [17]).

2 For us, $L(s, F)$ denotes a classical spinor $L$-function associated to $F$, and not to an automorphic representation corresponding to $F$; hence the shift in the argument in comparison to [22].
} 
Note that the first condition above automatically implies that $F$ is an eigenform of the local Hecke algebra at all primes not dividing $N_{2}$. For general $N_{1}, N_{2}$, there is no known explicit construction that generalises the classical Saito-Kurokawa lifts and exhausts the set of all P-CAP $F$ of level $\Gamma_{0}\left(N_{1}, N_{2}\right)$. It seems difficult then to directly prove Maass relations from construction. Even for $N_{1}=1$ this is already reflected in a comment made by Ibukiyama in [7] after providing the relations for a special subset of Fourier coefficients. In this work we are able to derive Maass relations using methods of representation theory.

Theorem 1 Let $N_{1}, N_{2}$ be positive integers, $N_{1} \mid N_{2}$, and let $F$ be a cuspidal Siegel modular form of weight $k$ and level $\Gamma_{0}\left(N_{1}, N_{2}\right)$ that is associated to a P-CAP representation. Let $a, b, c$ be integers such that $\operatorname{gcd}\left(a, b, c, N_{2}\right)=1, b^{2}-4 a c<0$ and $\left(\frac{b^{2}-4 a c}{p}\right)=-1$ for all $p \mid N_{1}$, and let $L$ be any positive integer whose all prime factors divide $\mathrm{N}_{2}$. Then

$$
a\left(F, L\left(\begin{array}{cc}
a & b / 2 \\
b / 2 & c
\end{array}\right)\right)=\sum_{r \mid \operatorname{gcd}(a, b, c)} r^{k-1} a\left(F, L\left(\begin{array}{cc}
\frac{a c}{r^{2}} & \frac{b}{2 r} \\
\frac{b}{2 r} & 1
\end{array}\right)\right) .
$$

In fact, theorem stated above is a corollary of a more general result (Theorem 4), where the condition on $\left(\frac{b^{2}-4 a c}{p}\right)$ is replaced with an exact condition on a certain ray class group. The simplification presented above follows from a connection between equivalence classes of binary quadratic forms and elements of suitable ray class groups; this is the topic of Sect. 4 and may be of independent interest.

A reader may find it surprising or unsatisfactory that, in contrary to the classical Maass relations, the right-hand side of the above equality contains $L$. However, there are modular forms for which this cannot be changed. Indeed, as Schmidt showed in [22], there exist paramodular forms that are Saito-Kurokawa lifts, and all their Fourier coefficients $a(F, T)$ have of course the $(2,2)$-entry of $T$ divisible by the level.

We should also mention that Ibukiyama's relations alluded to above, when restricted to $F$ with a trivial character, are included in our result. In particular, one of the assumptions there was that $F \in S_{k}^{(2)}\left(\Gamma_{0}(1, N)\right)$ has a non-zero Fourier Jacobi coefficient of index 1 ; this is not necessary in our construction.

Our work extends the method developed in [17] to Saito-Kurokawa lifts of higher levels. It bases on the relation satisfied by vectors in local Bessel models for P-CAP representations ([17, Theorem 2.1]) and the fact that certain values of a global Bessel period associated to a Siegel modular form $F$ can be expressed in terms of Fourier coefficients of $F$. We compute these values explicitly for Siegel modular forms invariant under the action of $\Gamma_{0}\left(N_{1}, N_{2}\right)$ with $N_{1} \mid N_{2}$, and combine it with the local-global relation (10) satisfied by Bessel periods to obtain a relation between Fourier coefficients (Theorem 3; generalisation of [9, Theorem 2.10]). Theorem 3 is a basis for our main result.

Another approach to obtain Maass relations for $F \in S_{k}^{(2)}(\Gamma)$ for some congruence subgroup $\Gamma$ of $\mathrm{Sp}_{4}(\mathbb{Q})$ might be to follow the aforementioned construction of SaitoKurokawa lifting due to Piatetski-Shapiro [12]. This was done by Ichino [8, Lemma 7.1] for $\Gamma=\mathrm{Sp}_{4}(\mathbb{Z})$ and very recently extended by Chen [2, Lemma 5.7] to $\Gamma=$ 
$\Gamma_{0}(1, N)$ with $N$ odd and square-free. In fact, their motivation to choose such an approach lied in establishing a formula for pullbacks of Saito-Kurokawa lifts and its application to algebraicity of central critical values of certain automorphic $L$-functions for $\mathrm{Sp}_{2} \times \mathrm{GL}_{2}$ and $\mathrm{GL}_{3} \times \mathrm{GL}_{2}$.

Throughout the paper we use the following notation.

- $\mathbb{N}, \mathbb{Z}, \mathbb{Q}, \mathbb{R}, \mathbb{C}$ stand for the natural, integer, rational, real and complex numbers, respectively; $\mathbb{Q}_{p}$ denotes the $p$-adic numbers and $\mathbb{Z}_{p}$ the $p$-adic integers, $\mathbb{A}$ stands for the adeles of $\mathbb{Q}$ and $\mathbb{A}_{f}:=\prod_{p<\infty}^{\prime} \mathbb{Q}_{p}$ the finite adeles; the set of invertible elements in a ring $R$ is denoted by $R^{\times}$;

- $M_{n}$ denotes the set of $n \times n$ matrices, whose identity element is $1_{n}$, and $i_{2}:=i 1_{2}$; we use the superscript $M_{n}^{\text {sym }}$ for symmetric matrices, and $M_{n}^{+}$for the matrices with positive determinant;

we distinguish a set

$$
\mathscr{P}_{n}:=\left\{T \in \frac{1}{2} M_{n}^{\text {sym }}(\mathbb{Z}): T \text { half-integral and positive definite }\right\},
$$

where half-integral means that $T$ has integers on the diagonal;

${ }^{t} T$ is the transpose of $T$ and $\operatorname{tr} T$ the trace of $T$;

$$
\text { cont }\left(\begin{array}{cc}
a & b / 2 \\
b / 2 & c
\end{array}\right):=\operatorname{gcd}(a, b, c), \quad \operatorname{disc}\left(\begin{array}{cc}
a & b / 2 \\
b / 2 & c
\end{array}\right):=b^{2}-4 a c
$$

are the content and the discriminant of the matrix $\left(\begin{array}{cc}a & b / 2 \\ b / 2 & c\end{array}\right)$;

- The letter $G$ will always stand for the group $\mathrm{GSp}_{4}$ defined as follows:

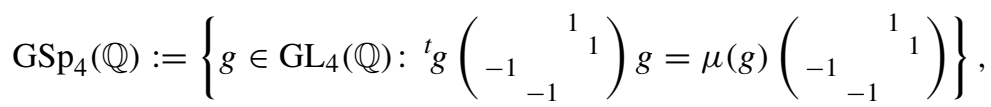

where $\mu(g) \in \mathbb{Q}^{\times}, \mathrm{Sp}_{4}(\mathbb{Q}):=\{g \in G(\mathbb{Q}): \mu(g)=1\}$; and for $n_{1} \leq n_{2}$ :

$$
I\left(n_{1}, n_{2}\right):=G\left(\mathbb{Z}_{p}\right) \cap\left(\begin{array}{llll}
\mathbb{Z}_{p} & p^{n_{1}} \mathbb{Z}_{p} & \mathbb{Z}_{p} & \mathbb{Z}_{p} \\
\mathbb{Z}_{p} & \mathbb{Z}_{p} & \mathbb{Z}_{p} & \mathbb{Z}_{p} \\
p^{n_{2}} \mathbb{Z}_{p} & p^{n_{2}} \mathbb{Z}_{p} & \mathbb{Z}_{p} & \mathbb{Z}_{p} \\
p^{n_{2}} \mathbb{Z}_{p} & p^{n_{2}} \mathbb{Z}_{p} & p^{n_{1}} \mathbb{Z}_{p} & \mathbb{Z}_{p}
\end{array}\right)
$$


- For $N=\prod_{p} p^{n_{p}}$ and $N_{1} \mid N_{2}$ having similar prime factorizations we define

$$
\begin{aligned}
& I_{N_{1}, N_{2}}:=\prod_{p<\infty} I\left(n_{1, p}, n_{2, p}\right) ; \\
& K_{N}^{*}:=\prod_{p<\infty}\left\{g \in \mathrm{GL}_{2}\left(\mathbb{Z}_{p}\right): g=\left(\begin{array}{l}
* \\
*
\end{array}\right) \bmod p^{n_{p}}\right\} ; \\
& \Gamma_{0}\left(N_{1}, N_{2}\right):=G(\mathbb{Q}) \cap G(\mathbb{R})^{+} I_{N_{1}, N_{2}}=\operatorname{Sp}_{4}(\mathbb{Z}) \cap\left(\begin{array}{llll}
\mathbb{Z} & N_{1} \mathbb{Z} & \mathbb{Z} & \mathbb{Z} \\
\mathbb{Z} & \mathbb{Z} & \mathbb{Z} & \mathbb{Z} \\
N_{2} \mathbb{Z} & N_{2} \mathbb{Z} & \mathbb{Z} & \mathbb{Z} \\
N_{2} \mathbb{Z} & N_{2} \mathbb{Z} & N_{1} \mathbb{Z} & \mathbb{Z}
\end{array}\right) ;
\end{aligned}
$$

- For $N \in \mathbb{N}$,

$$
\Gamma_{0}(N):=\mathrm{SL}_{2}(\mathbb{Z}) \cap\left(\begin{array}{cc}
\mathbb{Z} & \mathbb{Z} \\
N \mathbb{Z} & \mathbb{Z}
\end{array}\right), \quad \Gamma^{0}(N):=\mathrm{SL}_{2}(\mathbb{Z}) \cap\left(\begin{array}{c}
\mathbb{Z} N \mathbb{Z} \\
\mathbb{Z}
\end{array}\right)
$$

- For $N \in \mathbb{N}, X \in \mathbb{Z}$ we put

$$
\left(X, N^{\infty}\right):=\prod_{p \mid N} p^{\mathrm{ord}_{p} X}, \quad \text { where } \operatorname{ord}_{p} X:=\max \left\{n \in \mathbb{Z}: p^{n} \mid X\right\}
$$

and $N^{\infty}$ denotes a formal number such that $N^{l} \mid N^{\infty}$ for all $l \in \mathbb{Z}^{+}$;

$$
p^{k} \| N \quad \text { means } \quad k=\operatorname{ord} p
$$

$\left(\frac{X}{p}\right)$ denotes the Legendre symbol.

\section{Preliminaries}

Let $\pi=\otimes_{p} \pi_{p}$ be an irreducible automorphic cuspidal representation of $G(\mathbb{A})$ with trivial central character and such that $\pi_{\infty}=\mathscr{E}(k, k)$, the lowest weight representation of scalar minimal K-type of weight $k$ (see [13]). Let $\Phi$ be an automorphic form in the space of $\pi$ and let $\phi_{\infty}$ be a lowest weight vector of $\pi_{\infty}$. This means that

$$
\Phi\left(g k_{\infty}\right)=j\left(k_{\infty}, i_{2}\right)^{-k} \Phi(g) \quad \text { for all } k_{\infty} \in K_{\infty}, g \in G(\mathbb{A})
$$

where

(i) $K_{\infty}$ is the standard maximal compact subgroup of $G(\mathbb{R})^{+}$,

(ii) $j\left(\left(\begin{array}{ll}A & B \\ C & D\end{array}\right), i_{2}\right)=\operatorname{det}\left(C i_{2}+D\right)$.

Furthermore, assume that there are integers $N_{1}, N_{2}$ with $N_{1} \mid N_{2}$ such that $\Phi$ is right invariant by $I_{N_{1}, N_{2}}$. 
Define

$$
F(Z):=\Phi(g) j\left(g, i_{2}\right)^{k} \mu(g)^{-k},
$$

where $g \in G(\mathbb{R})$ is such that $g \cdot i_{2}=Z$ and $\left(\begin{array}{cc}A & B \\ C & D\end{array}\right) \cdot i_{2}:=\left(A i_{2}+B\right)\left(C i_{2}+D\right)^{-1}$. Such a function $F$ is holomorphic and satisfies

$$
\left.F\right|_{k} \gamma(Z):=\mu(\gamma)^{k} j(\gamma, Z)^{-k} F(\gamma \cdot Z)=F(Z) \quad \text { for every } \gamma \in \Gamma_{0}\left(N_{1}, N_{2}\right)
$$

it is a cuspidal Siegel modular form of degree 2, level $\Gamma_{0}\left(N_{1}, N_{2}\right)$ and weight $k$ that is an eigenform of the local Hecke algebra at all primes $p \nmid N_{2} \cdot{ }^{3}$ It follows that $F$ admits a unique Fourier expansion

$$
F(Z)=\sum_{T \in \mathscr{P}_{2}} a(F, T) e(\operatorname{tr}(T Z)), \text { where } e(x):=e^{2 \pi i x},
$$

and its Fourier coefficients satisfy

$$
a(F, T)=a\left(F,{ }^{t} A T A\right) \quad \text { for every } A \in \Gamma^{0}\left(N_{1}\right) .
$$

Observe that the correspondence (2) is bijective in a sense that to any Siegel cusp form $F$ that satisfies the above conditions and gives rise to an irreducible representation we can attach an automorphic form $\Phi$, the adelisation of $F$, via

$$
\Phi(g):=\left.F\right|_{k} g_{\infty}\left(i_{2}\right), \quad g=g_{\mathbb{Q}} g_{\infty} g_{0} \in G(\mathbb{Q}) G(\mathbb{R})^{+} I_{N_{1}, N_{2}}=G(\mathbb{A}) .
$$

\section{Local and global Bessel models}

Throughout this section $F$ is a non-archimedean local field of characteristic zero, $\mathfrak{o}$ its ring of integers, $\mathfrak{p}$ the maximal ideal of $\mathfrak{o}, \varpi$ a generator of $\mathfrak{p}$, and $q$ the cardinality of the residue field $\mathfrak{o} / \varpi \mathfrak{o}$. For our global application we will only need $F=\mathbb{Q}_{p}$, but because of a deeper analysis of an ideal class group in the next section, we would like to describe a torus $T$ in more detail.

\subsection{Local Bessel models for $\mathrm{GSp}_{4}$}

We recall the definition of the Bessel model following the exposition of Furusawa [6] and Pitale and Schmidt [16]. Let $S \in M_{2}^{\text {sym }}(F)$ be such that $d=\operatorname{disc} S=-4 \operatorname{det} S \neq$ 0 . For

$$
S=\left(\begin{array}{cc}
a & b / 2 \\
b / 2 & c
\end{array}\right)
$$

\footnotetext{
${ }^{3}$ In fact, these functions, coming from irreducible representations, span the space of Siegel cusp forms of degree 2 , level $\Gamma_{0}\left(N_{1}, N_{2}\right)$ and weight $k$. 
we define the element

$$
\xi=\xi_{S}=\left(\begin{array}{cc}
b / 2 & c \\
-a & -b / 2
\end{array}\right)
$$

and denote by $F(\xi)$ a two-dimensional $F$-algebra generated by $1_{2}$ and $\xi$. Note that

$$
\xi^{2}=\left(\begin{array}{ll}
\frac{d}{4} & \\
& \frac{d}{4}
\end{array}\right)
$$

Depending whether $d$ is a square in $F^{\times}$or not, $F(\xi)$ is isomorphic either to $L=F \oplus F$ or to the field $L=F(\sqrt{d})$ via

$$
x 1_{2}+y \xi \longmapsto \begin{cases}x+y \frac{\sqrt{d}}{2}, & d \notin\left(F^{\times}\right)^{2}, \\ \left(x+y \frac{\sqrt{d}}{2}, x-y \frac{\sqrt{d}}{2}\right), & d \in\left(F^{\times}\right)^{2} .\end{cases}
$$

The determinant map on $F(\xi)$ corresponds to the norm map $N_{L / F}(z)=z \bar{z}$ on $L$, where $z \mapsto \bar{z}$ is the usual involution on $L$ fixing $F$. We define the Legendre symbol as

$$
\left(\frac{L}{\mathfrak{p}}\right)= \begin{cases}-1 & \text { if } L / F \text { is an unramified field extension } \\ 0 & \text { if } L / F \text { is a ramified field extension, } \\ 1 & \text { if } L=F \oplus F\end{cases}
$$

If $L$ is a field, denote by $\mathfrak{o}_{L}, \mathfrak{p}_{L}, \varpi_{L}$ the ring of integers, the maximal ideal of $\mathfrak{o}_{L}$ and a fixed choice uniformiser in $\mathfrak{o}_{L}$, correspondingly. If $L=F \oplus F$, let $\mathfrak{o}_{L}=\mathfrak{o} \oplus \mathfrak{o}$, $\varpi_{L}=(\varpi, 1)$. Define an ideal $\mathfrak{P}:=\mathfrak{p o}_{L}$ in $\mathfrak{o}_{L}$; note that $\mathfrak{P}=\mathfrak{p}_{L}$ is prime only if $\left(\frac{L}{\mathfrak{p}}\right)=-1$, otherwise $\mathfrak{P}=\mathfrak{p}_{L}^{2}$ if $\left(\frac{L}{\mathfrak{p}}\right)=0$ and $\mathfrak{P}=\mathfrak{p} \oplus \mathfrak{p}$ if $\left(\frac{L}{\mathfrak{p}}\right)=1$.

We define a subgroup $T=T_{S}$ of $\mathrm{GL}_{2}$ by

$$
T(F)=\left\{g \in \mathrm{GL}_{2}(F):{ }^{t} g S g=\operatorname{det} g \cdot S\right\} .
$$

It is not hard to verify that $T(F)=F(\xi)^{\times}$, so that $T(F) \cong L^{\times}$. We identify $T(F)$ with $L^{\times}$via (4). We can consider $T$ as a subgroup of $G$ via

$$
T \ni g \longmapsto\left(\begin{array}{cc}
g & 0 \\
0 & \operatorname{det} g \cdot{ }^{t} g^{-1}
\end{array}\right) \in G
$$

Let us denote by $U$ the subgroup of $G$ defined by

$$
U=\left\{u(X)=\left(\begin{array}{cc}
1_{2} & X \\
0 & 1_{2}
\end{array}\right):{ }^{t} X=X\right\}
$$

and finally let $R$ be the subgroup of $G$ defined by $R=T U$. 
We fix a non-trivial additive character $\psi$ of $F$ such that $\psi$ is trivial on $\mathfrak{o}$, but non-trivial on $\mathfrak{p}^{-1}$, and define the character $\theta=\theta_{S}$ on $U(F)$ by

$$
\theta(u(X)):=\psi(\operatorname{tr}(S X))
$$

Let $\Lambda$ be a character of $T(F)$ such that $\left.\Lambda\right|_{F^{\times}}=1$. Denote by $\Lambda \otimes \theta$ the character of $R(F)$ defined by $(\Lambda \otimes \theta)(t u):=\Lambda(t) \theta(u)$ for $t \in T(F), u \in U(F)$.

Let $\pi$ be an irreducible admissible representation of the group $G(F)$ with trivial central character. We say that such a $\pi$ has a local Bessel model of type $(\Lambda, \theta)$ if $\pi$ is isomorphic to a subrepresentation of the space of all locally constant functions $B$ on $G(F)$ satisfying the local Bessel transformation property

$$
B(r g)=(\Lambda \otimes \theta)(r) B(g) \quad \text { for all } r \in R(F) \text { and } g \in G(F) \text {. }
$$

It is known by $[11,18]$ that if a local Bessel model exists, then it is unique. If the local Bessel model for $\pi$ exists, we denote it by $\mathscr{B}_{\Lambda, \theta}^{\pi}$. In this case, we fix a (unique up to scalar) isomorphism of representations $\pi \rightarrow \mathscr{B}_{\Lambda, \theta}^{\pi}$ and denote the image of any $\phi \in \pi$ by $B_{\phi}$. In fact, one can always assume that the local Bessel model is defined with respect to the matrix $S$ whose entries $a, b, c$ and the discriminant $d=b^{2}-4 a c$ satisfy the following conditions:

- $a, b \in \mathfrak{o}$ and $c \in \mathfrak{o}^{\times}$.

- If $d \notin\left(F^{\times}\right)^{2}$, then $d$ is a generator of the discriminant of $L / F$.

- If $d \in\left(F^{\times}\right)^{2}$, then $d \in \mathfrak{o}^{\times}$.

Indeed, it is easy to check that for any $A \in \mathrm{GL}_{2}(F)$ and $\alpha \in F^{\times}$the map

$$
B(g) \longmapsto B\left(\left(\begin{array}{c}
A \\
\alpha^{-1 t} A^{-1}
\end{array}\right) g\right), \quad g \in G(F),
$$

defines an isomorphism between local Bessel models of types $\left(\Lambda, \theta_{S}\right)$ and $\left(\Lambda^{\prime}, \theta_{S^{\prime}}\right)$, where $S^{\prime}=\alpha^{t} A S A$ and $\Lambda^{\prime}\left(t^{\prime}\right)=\Lambda\left(A t^{\prime} A^{-1}\right)$ is a character of $T_{S^{\prime}}(F)=A^{-1} T_{S}(F) A$. A bit more work ${ }^{4}$ leads to the matrix $S$ satisfying the conditions (7).

\subsection{Sugano's formula}

We now investigate more closely the case when $\pi$ is spherical, that is, $\pi$ has a nonzero $G(\mathfrak{o})$-invariant vector. Such a representation is a constituent of a representation parabolically induced from an unramified character $\gamma$ of the Borel subgroup of $G(F)$. The values of the character $\gamma$ at the matrices

$$
\left(\begin{array}{llll}
\varpi & & & \\
& \varpi & & \\
& & 1 & 1
\end{array}\right),\left(\begin{array}{lllll}
\varpi & & & \\
& 1 & & \\
& & 1 & \\
& & & \varpi
\end{array}\right),\left(\begin{array}{cccc}
1 & & & \\
& 1 & & \\
& & \varpi & \\
& & & \varpi
\end{array}\right),\left(\begin{array}{llll}
1 & & & \\
& \varpi & & \\
& & \varpi & 1
\end{array}\right)
$$

\footnotetext{
${ }^{4}$ See Lemma 1.1 in a long version of Pitale, Schmidt, Bessel models for GSp(4): Siegel vectors of squarefree level available at http://www.math.unt.edu/ schmidt/. 
are called the Satake parameters of $\pi$ and determine the isomorphism class of $\pi$.

Throughout this section we assume the following:

(i) $\pi$ is a spherical representation of $G(F)$,

(ii) $S=\left(\begin{array}{cc}a & b / 2 \\ b / 2 & c\end{array}\right)$ with $a, b, c$ satisfying the conditions (7),

(iii) $\theta=\theta_{S}$ is the character of $U(F)$ as in (6),

(iv) $\Lambda$ is a character of $T(F)$ that is invariant under the subgroup

$$
T(n):=T(F) \cap\left\{g \in \mathrm{GL}_{2}(\mathfrak{o}): g=\left(\begin{array}{c}
\lambda \\
\lambda
\end{array}\right) \bmod \mathfrak{p}^{n}, \lambda \in \mathfrak{o}^{\times}\right\},
$$

for some non-negative integer $n$.

The next lemma shows equivalent ways of writing the group $T(n)$. Thanks to this, our definition coincides with the one used in [16,17].

Lemma 1 The group $T(n)$ defined above is isomorphic to each of the following:

$$
\begin{aligned}
& T(F) \cap\left\{g \in \mathrm{GL}_{2}(\mathfrak{o}): g=\left(\begin{array}{l}
* \\
* *
\end{array}\right) \bmod \mathfrak{p}^{n}\right\}, \\
& T(F) \cap\left\{g \in \mathrm{GL}_{2}(\mathfrak{o}): g=\left(\begin{array}{c}
* \\
*
\end{array}\right) \bmod \mathfrak{p}^{n}\right\}
\end{aligned}
$$

and (under the isomorphism $T(F) \cong L^{\times}$)

$$
\mathfrak{o}^{\times}\left(1+\mathfrak{P}^{n}\right) \cap \mathfrak{o}_{L}^{\times}
$$

Moreover, every character of $T(F)$ that is trivial on $\mathfrak{o}^{\times}$is trivial on $T(n)$ for $n$ big enough.

Proof By [14, Lemma 3.1.1], $\mathfrak{o}_{L}=\mathfrak{o}+\mathfrak{o} \xi_{0}$, where

$$
\xi_{0}= \begin{cases}\frac{-b+\sqrt{d}}{2} & \text { if } L \text { is a field } \\ \left(\frac{-b+\sqrt{d}}{2}, \frac{-b-\sqrt{d}}{2}\right) & \text { if } L=F \oplus F\end{cases}
$$

Therefore by the identification (4),

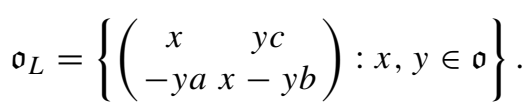

Hence, under the assumptions (7) and via the isomorphism $T(F) \cong L^{\times}$, the group $T(\mathfrak{o}):=T(F) \cap \mathrm{GL}_{2}(\mathfrak{o})$ is isomorphic to $\mathfrak{o}_{L}^{\times}$. Moreover, for any $\lambda \in \mathfrak{o}^{\times}$:

$$
\lambda\left(1+\mathfrak{P}^{n}\right) \cap \mathfrak{o}_{L}^{\times} \cong \lambda\left(1_{2}+\mathfrak{p}^{n} \mathfrak{o}_{L}\right) \cap T(\mathfrak{o}),
$$


and thus

$$
\mathfrak{o}^{\times}\left(1+\mathfrak{P}^{n}\right) \cap \mathfrak{o}_{L}^{\times} \cong T(n)
$$

Assume now that $g \in T(F) \cap \mathrm{GL}_{2}(\mathfrak{o})$ is congruent to $(\underset{* *}{*}) \bmod \mathfrak{p}^{n}$. We already know that $g$ must be of the form $x 1_{2}+y\left(\begin{array}{c}c \\ -a-b\end{array}\right)$ with $x, y \in \mathfrak{o}$. However, because $c \in \mathfrak{o}^{\times}$, we have $y \in \mathfrak{p}^{n} \mathfrak{o}$, and thus $g=\left({ }^{x} x\right) \bmod \mathfrak{p}^{n}$, which means that $g \in T(n)$. The other inclusions are clear.

To prove the last assertion we use the isomorphism ( $\star$ ). Because $\mathfrak{o}^{\times}$is compact and $\left\{\lambda\left(1+\mathfrak{P}^{n}\right) \cap \mathfrak{o}_{L}^{\times}: n \in \mathbb{N}\right\}$ gives a set of neighbourhoods of each $\lambda \in \mathfrak{o}^{\times}$in $\mathfrak{o}_{L}^{\times}$, so if $\Lambda$ is trivial on $\mathfrak{o}^{\times}$, it must be trivial on $\left\{\lambda\left(1+\mathfrak{P}^{n}\right) \cap \mathfrak{o}_{L}^{\times}: \lambda \in \mathfrak{o}^{\times}\right\}$for $n$ big enough.

Definition 1 The smallest integer $n$ for which $\Lambda$ is $T(n)$-invariant or, equivalently,

$$
\min \left\{n \geq 0:\left.\Lambda\right|_{\mathfrak{o}^{\times}}\left(1+\mathfrak{P}^{n}\right) \cap \mathfrak{o}_{L}^{\times}=1\right\}
$$

will be denoted by $c(\Lambda)$.

Thanks to Roberts and Schmidt, [19, Theorem 6.2.2], we know exactly for which characters $\Lambda$ the representation $\pi$ admits a local Bessel model $\mathscr{B}_{\Lambda, \theta}^{\pi}$. For example, if $\pi$ is an irreducible spherical principal series representation (type I there), such a local Bessel model exists for all $\Lambda$. This model contains a unique (up to multiples) $G(\mathfrak{o})$ invariant vector, which we will denote by $B_{\pi}^{(0)}(\Lambda, \theta)$ or by $B_{\pi}^{(0)}$. Moreover, Sugano provided an explicit formula for the values of $B_{\pi}^{(0)}$ at

$$
h(l, m):=\left(\begin{array}{cccc}
\varpi^{l+2 m} & & & \\
& \varpi^{l+m} & & \\
& & 1 & \\
& & & \varpi^{m}
\end{array}\right), \quad l, m \in \mathbb{Z}, m \geq 0 .
$$

Theorem 2 (Sugano [23]) Assume (i)-(iv), and let $\Lambda$ be such that the local Bessel model $\mathscr{B}_{\Lambda, \theta}^{\pi}$ exists. Then

1. $B_{\pi}^{(0)}(h(l, m))=0$ if $l<0$ or $m<c(\Lambda)$.

2. $B_{\pi}^{(0)}(h(0, c(\Lambda))) \neq 0$.

Moreover,

$$
\sum_{l, m \geq 0} B_{\pi}^{(0)}(h(l, m)) x^{m} y^{l}=\frac{H(x, y)}{P(x) Q(y)},
$$

where $P(x), Q(y)$ and $H(x, y)$ are (explicit) polynomials depending on $F, L, \Lambda$ and the Satake parameters of $\pi$.

Because of the above theorem, if the local Bessel model $\mathscr{B}_{\Lambda, \theta}^{\pi}$ exists, we can and will henceforth normalise $B_{\pi}^{(0)}$ so that $B_{\pi}^{(0)}(h(0, c(\Lambda)))=1$. 


\subsection{Global Bessel models for $\mathrm{GSp}_{4}$}

Let $d$ be a fundamental discriminant and

$$
S(d)=\left(\begin{array}{cc}
a & b / 2 \\
b / 2 & c
\end{array}\right):= \begin{cases}\left(\begin{array}{cc}
\frac{-d}{4} & 0 \\
0 & 1
\end{array}\right) & \text { if } d \equiv 0 \quad(\bmod 4) \\
\left(\begin{array}{cc}
\frac{1-d}{4} & \frac{1}{2} \\
\frac{1}{2} & 1
\end{array}\right) & \text { if } d \equiv 1 \quad(\bmod 4) .\end{cases}
$$

Let groups $T, U, R$ be as above and let $\psi$ be a fixed non-trivial character of $\mathbb{A} / \mathbb{Q}$. We define the character $\theta=\theta_{S}$ on $U(\mathbb{A})$ by $\theta(u(X)):=\psi(\operatorname{tr}(S X))$. Let $\Lambda$ be a character of $T(\mathbb{A}) / T(\mathbb{Q})$ such that $\left.\Lambda\right|_{\mathbb{A}^{\times}}=1$, and denote by $\Lambda \otimes \theta$ the character of $R(\mathbb{A})$ defined by $(\Lambda \otimes \theta)(t u):=\Lambda(t) \theta(u)$ for $t \in T(\mathbb{A}), u \in U(\mathbb{A})$.

Let $\pi$ be an irreducible cuspidal automorphic representation of $G(\mathbb{A})$ with trivial central character and $V_{\pi}$ be its space of automorphic forms. For $\Phi \in V_{\pi}$, we define a function $B_{\Phi}$ on $G(\mathbb{A})$ by

$$
B_{\Phi}(g)=\int_{\mathbb{A}^{\times} R(\mathbb{Q}) \backslash R(\mathbb{A})}(\Lambda \otimes \theta)(r)^{-1} \Phi(r g) d r .
$$

The $\mathbb{C}$-vector space of functions on $G(\mathbb{A})$ spanned by $\left\{B_{\Phi}: \Phi \in V_{\pi}\right\}$ is called the global Bessel space of type $(\Lambda, \theta)$ for $\pi$, and its vectors are called Bessel periods; it is invariant under the regular action of $G(\mathbb{A})$, and when the space is non-zero, the corresponding representation is a model of $\pi$, which we call a global Bessel model of type $(\Lambda, \theta)$.

For $\Phi \in V_{\pi}$ and a symmetric matrix $S \in M_{2}(\mathbb{Q})$, we define the Fourier coefficient

$$
\Phi_{S, \psi}(g)=\int_{M_{2}^{\mathrm{sym}}(\mathbb{Q}) \backslash M_{2}^{\mathrm{sym}}(\mathbb{A})} \psi^{-1}(\operatorname{tr}(S X)) \Phi\left(\left(\begin{array}{ll}
1_{2} & X \\
& 1_{2}
\end{array}\right) g\right) d X, \quad g \in G(\mathbb{A})
$$

For brevity we will often shorten $\Phi_{S, \psi}$ to $\Phi_{S}$.

\section{Ray class groups and ${ }^{0}(\mathrm{~N})$-equivalence}

In the next section we will describe a relation between Fourier coefficients of Siegel modular forms of degree 2 and values of Bessel periods at the matrices similar to $h_{p}(l, m)$. As we shall see, these values are equal to sums of Fourier coefficients indexed via elements of a ray class group. The question is whether all the coefficients of fixed content and discriminant, up to equivalence, occur in such sum. It will be the subject of this section.

Recall that each coefficient $a(F, T)$ may be characterised according to content and discriminant of $T$, and each discriminant may be written as $d M^{2} L^{2}$, where $d$ is a 
fundamental discriminant ${ }^{5}$ and $L$ content of the matrix $T$. From now on $d$ will denote a negative fundamental discriminant.

In view of the relation (3) satisfied by Fourier coefficients of $F$, we define the set

$$
H\left(d M^{2}, L ; \Gamma^{0}(N)\right):=\left\{T \in \mathscr{P}_{2}: \operatorname{disc} T=d L^{2} M^{2}, \text { cont } T=L\right\} / \sim,
$$

where

$$
T \sim T^{\prime} \quad \Longleftrightarrow \quad T^{\prime}={ }^{t} A T A \text { for some } A \in \Gamma^{0}(N)
$$

It is well known that when $M=N=1$, the set $H\left(d, L ; \Gamma^{0}(1)\right)$ is isomorphic to the ideal class group of $\mathbb{Q}(\sqrt{d})$. As we shall see, when $M, N>1$ the situation is more complicated. In [17], Pitale et al. found a bijection between $H\left(d M^{2}, L ; \Gamma^{0}(1)\right)$ and a certain ray class group of $\mathbb{Q}(\sqrt{d})$, which we will call later $\mathrm{Cl}_{d}(M)$. We are going to extend their result to $N>1$.

\subsection{Construction of an endomorphism}

Fix positive integers $M, N$ and a negative fundamental discriminant $d$. Let $S(d)$ and $T=T_{S(d)}$ be as in Sect. 3.

Definition 2 For $N=\prod_{p} p^{n_{p}}$ define

$$
T_{N}:=\prod_{p<\infty} T\left(n_{p}\right) \text { and } \mathrm{Cl}_{d}(N):=T(\mathbb{A}) / T(\mathbb{Q}) T(\mathbb{R}) T_{N}
$$

where $T\left(n_{p}\right) \subseteq T\left(\mathbb{Q}_{p}\right)$ is as in Sect. 3.2 and by $T(0)$ we mean the maximal compact subgroup $T\left(\mathbb{Z}_{p}\right):=T\left(\mathbb{Q}_{p}\right) \cap \mathrm{GL}_{2}\left(\mathbb{Z}_{p}\right)$ of $T\left(\mathbb{Q}_{p}\right)$.

Because of the isomorphism described in Lemma 1, we may view $\mathrm{Cl}_{d}(N)$ as a ray class group of $\mathbb{Q}(\sqrt{d})$.

Basing on the argument of [17], we will now describe a certain map from $\mathrm{Cl}_{d}\left(N^{\prime}\right)$ to $H\left(d M^{2}, L ; \Gamma^{0}(N)\right)$, where $N^{\prime}$ is any integer divisible by $M N$.

Let $c \in \mathrm{Cl}_{d}\left(N^{\prime}\right)$ and let $t_{c} \in T(\mathbb{A})$ be a representative for $c$ so that $t_{c} \in$ $\prod_{p<\infty} T\left(\mathbb{Q}_{p}\right)$. By strong approximation we can write $t_{c}=\gamma_{c} m_{c} \kappa_{c}$, where $\gamma_{c} \in$ $\mathrm{GL}_{2}(\mathbb{Q}), m_{c} \in \mathrm{GL}_{2}(\mathbb{R})^{+}$and $\kappa_{c} \in K_{N^{\prime}}^{*}$. Also, denote by $\left(\gamma_{c}\right)_{f}$ the finite part of $\gamma_{c}$ when considered as an element of $\mathrm{GL}_{2}(\mathbb{A})$. Since $t_{c} \in \prod_{p<\infty} T\left(\mathbb{Q}_{p}\right),\left(\gamma_{c}\right)_{f}=\gamma_{c} m_{c}$ as elements of $\mathrm{GL}_{2}(\mathbb{A})$. Let

$$
S_{c}:=\operatorname{det}\left(\gamma_{c}\right)^{-1 t} \gamma_{c} S(d) \gamma_{c}
$$

\footnotetext{
${ }^{5}$ Recall that $d$ is a fundamental discriminant if $d$ is square-free and $d \equiv 1(\bmod 4)$ or $d=4 d^{\prime}, d^{\prime}$ squarefree and $d^{\prime} \equiv 2,3(\bmod 4)$. Or, equivalently, if $d=1$ or $d$ is the discriminant of a quadratic number field.
} 
it is a positive definite, half-integral, symmetric matrix of discriminant $d$ and content 1 (cf. [6], p. 209). In this way,

$$
\phi_{L, M}(c):=L\left(\begin{array}{ll}
M & 1
\end{array}\right) S_{c}\left(\begin{array}{ll}
M & 1
\end{array}\right)
$$

is a matrix of discriminant $d M^{2} L^{2}$ and content $L$.

Note that the matrices $\phi_{L, M}(c)$ constructed above are not uniquely defined, as they depend on the choice of $t_{c}$ and $\kappa_{c}$. However, the definition is correct for $\Gamma^{0}(N)$ equivalence classes and, actually, by an easy adaptation of the proof of Proposition 5.3, [17], we obtain the following.

Proposition 1 Assume that $M N \mid N^{\prime}$. Then the map $\tilde{\phi}_{L, M}=\tilde{\phi}_{L, M ; N^{\prime}}$ from $\mathrm{Cl}_{d}\left(N^{\prime}\right)$ to $H\left(d M^{2}, L ; \Gamma^{0}(N)\right)$, sending $c$ to $\phi_{L, M}(c)$ is well-defined. Moreover, if $N^{\prime}=M N$, $\tilde{\phi}_{L, M ; N^{\prime}}$ is injective.

\subsection{The image of $\tilde{\phi}_{L, M}$}

We start with the observation that the image of the map

$$
\tilde{\phi}_{L, M}: \mathrm{Cl}_{d}\left(N^{\prime}\right) \rightarrow H\left(d M^{2}, L ; \Gamma^{0}(N)\right), \quad c \mapsto \phi_{L, M}(c)
$$

constructed above does not depend on $N^{\prime}$, but only on $M N$.

Lemma 2 Let $M N\left|N_{1}^{\prime}\right| N_{2}^{\prime}$, and let $\rho: \mathrm{Cl}_{d}\left(N_{2}^{\prime}\right) \rightarrow \mathrm{Cl}_{d}\left(N_{1}^{\prime}\right)$ be the natural projection. Then the following diagram is commutative:

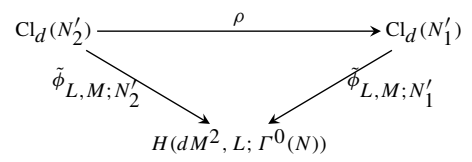

Proof This follows by construction. Let $c \in \mathrm{Cl}_{d}\left(N_{1}^{\prime}\right)$ and $c_{1}, c_{2}, \ldots, c_{t}$ be the elements of $\mathrm{Cl}_{d}\left(N_{2}^{\prime}\right)$ that the map $\rho$ sends to $c$. Choose distinct $i, j \in\{1,2, \ldots, t\}$. We will show that $\phi_{L, M}\left(c_{i}\right)$ and $\phi_{L, M}\left(c_{j}\right)$ are $\Gamma^{0}(N)$-equivalent. For this it suffices to find $\gamma \in \Gamma^{0}(N)$ such that $\gamma_{c_{i}}\left(M_{1}^{M}\right)=\gamma_{c_{j}}\left(M_{1}\right) \gamma$. Denote by $\left(\gamma_{c_{i}}\right)_{p}$ the image of $\gamma_{c_{i}}$ in $\mathrm{GL}_{2}\left(\mathbb{Z}_{p}\right)$, when embedded diagonally. Since $c_{i}, c_{j}$ map to $c,\left(\gamma_{c_{i}}\right)_{p} T\left(\operatorname{or} d_{p}\left(N_{1}^{\prime}\right)\right)=$ $\left(\gamma_{c_{j}}\right)_{p} T\left(\operatorname{ord} d_{p}\left(N_{1}^{\prime}\right)\right)$ for all primes $p \mid N_{1}^{\prime}$ or $p \nmid \frac{N_{2}^{\prime}}{N_{1}^{\prime}}$. Hence, for each of those primes there exists $g_{p} \in T\left(\operatorname{or} d_{p}\left(N_{1}^{\prime}\right)\right)$ such that $\left(\gamma_{c_{i}}\right)_{p}=\left(\gamma_{c_{j}}\right)_{p} g_{p}$. Note that we can choose $\gamma_{c_{i}}$ and $\gamma_{c_{j}}$ in such a way that $\gamma_{c_{i}} T(\mathbb{Q}) T(\mathbb{R}) \prod_{p} T\left(\mathbb{Z}_{p}\right)=\gamma_{c_{j}} T(\mathbb{Q}) T(\mathbb{R}) \prod_{p} T\left(\mathbb{Z}_{p}\right)$. Hence, for primes $p \mid \frac{N_{2}^{\prime}}{N_{1}^{\prime}}, g_{p}:=\left(\gamma_{c_{j}}^{-1}\right)_{p}\left(\gamma_{c_{i}}\right)_{p}$ is still in $T\left(\mathbb{Z}_{p}\right)$. This shows that $g:=$ $\gamma_{c_{j}}^{-1} \gamma_{c_{i}} \in \Gamma^{0}(M N)$. Now it's easy to check that $\gamma:=\left(\begin{array}{ll}1 / M_{1} & \end{array}\right) g\left(\begin{array}{ll}M_{1} & \end{array}\right)$ gives a desired $\Gamma^{0}(N)$-equivalence.

We denote

$$
H_{1}\left(d M^{2}, L ; \Gamma^{0}(N)\right):=\operatorname{im}\left(\tilde{\phi}_{L, M}: \mathrm{Cl}_{d}(M N) \rightarrow H\left(d M^{2}, L ; \Gamma^{0}(N)\right)\right) .
$$


Remark 1 The map $\tilde{\phi}_{L, M}$ from $\mathrm{Cl}_{d}(M N)$ to $H_{1}\left(d M^{2}, L ; \Gamma^{0}(N)\right)$ is a bijection. In this way the set $H_{1}\left(d M^{2}, L ; \Gamma^{0}(N)\right)$ acquires a natural group structure that makes it isomorphic to $\mathrm{Cl}_{d}(M N)$. Hence if $\Lambda$ is any character of $\mathrm{Cl}_{d}(M N)$, then we can naturally think of $\Lambda$ as a character of $H_{1}\left(d M^{2}, L ; \Gamma^{0}(N)\right)$.

In the next section we will naturally encounter sums like

$$
\sum_{c \in \mathrm{Cl}_{d}\left(N^{\prime}\right)} \Lambda^{-1}(c) a\left(F, \phi_{L, M}(c)\right)
$$

for a character $\Lambda$ of $\mathrm{Cl}_{d}\left(N^{\prime}\right)$. Observe that, if we denote by $\rho$ a natural projection from $\mathrm{Cl}_{d}\left(N^{\prime}\right)$ to $\mathrm{Cl}_{d}(M N)$, we have the following useful fact:

$$
\begin{aligned}
& \sum_{c \in \mathrm{Cl}_{d}\left(N^{\prime}\right)} \Lambda^{-1}(c) a\left(F, \phi_{L, M}(c)\right) \\
& =\sum_{c \in \mathrm{Cl}_{d}(M N)} a\left(F, \phi_{L, M}(c)\right) \sum_{\substack{\tilde{c} \in \mathrm{Cl}_{d}\left(N^{\prime}\right) \\
\rho(\tilde{c})=c}} \Lambda^{-1}(\tilde{c}) \\
& = \begin{cases}0 & \text { if } C(\Lambda) \nmid M N, \\
\frac{\left|\mathrm{Cl}_{d}\left(N^{\prime}\right)\right|}{\mid \mathrm{Cl}_{d}(M N \mid)} \sum_{T \in H_{1}\left(d M^{2}, L ; \Gamma^{0}(N)\right)} \Lambda^{-1}(T) a(F, T) & \text { if } C(\Lambda) \mid M N,\end{cases}
\end{aligned}
$$

where $C(\Lambda):=\prod_{p} p^{c\left(\Lambda_{p}\right)}$ is the smallest integer such that $\left.\Lambda\right|_{T_{C(\Lambda)}}=1$.

Let us now try to describe more accurately the set $H_{1}\left(d M^{2}, L ; \Gamma^{0}(N)\right)$. This will give us information on the coefficients occurring in the sum above.

Lemma 3 Suppose that $S^{\prime}=\left(\begin{array}{cc}a^{\prime} & b^{\prime} / 2 \\ b^{\prime} / 2 & c^{\prime}\end{array}\right)$ is a matrix of discriminant $d M^{2} L^{2}$ and content $L, \xi_{S^{\prime}}=\left(\begin{array}{cc}b^{\prime} / 2 & c^{\prime} \\ -a^{\prime} & -b^{\prime} / 2\end{array}\right)$. Let $E\left(S^{\prime}\right)$ be the subgroup of $\mathrm{SL}_{2}(\mathbb{Z})$ defined as

$$
E\left(S^{\prime}\right):=\left\{g \in \mathrm{SL}_{2}(\mathbb{Z}): g^{t} S^{\prime} g=S^{\prime}\right\} .
$$

Then

1. If $d \neq-4,-3$, or if $M>1$, then $E\left(S^{\prime}\right)=\left\{ \pm 1_{2}\right\}$.

2. If $(d, M)=(-4,1)$, then $E\left(S^{\prime}\right)=\left\{ \pm 1_{2}, \pm \xi_{S^{\prime}}\right\}$.

3. If $(d, M)=(-3,1)$, then $E\left(S^{\prime}\right)=\left\{ \pm 1_{2}, \pm\left(\frac{1}{2} 1_{2}+\xi_{S^{\prime}}\right), \pm\left(-\frac{1}{2} 1_{2}+\xi_{S^{\prime}}\right)\right\}$.

Proof Note that $E\left(S^{\prime}\right)=\left\{g \in T_{S^{\prime}}(\mathbb{Q}) \cap \mathrm{SL}_{2}(\mathbb{Z})\right.$ : det $\left.g=1\right\}$ and it doesn't depend on the content of $S^{\prime}$. We may assume then that $L=1$. A discussion at the beginning of Sect. 3.1 applies also when disc $S^{\prime}=d M^{2}$ and $F=\mathbb{Q}$, i.e. there is an identification

$$
T_{S^{\prime}}(\mathbb{Q})=\mathbb{Q}\left(\xi_{S^{\prime}}\right)^{\times} \ni x+y \xi_{S^{\prime}} \longmapsto x+y \frac{\sqrt{\operatorname{det} \xi_{S^{\prime}}}}{2}=x+y \frac{M \sqrt{d}}{2} \in \mathbb{Q}(\sqrt{d}) .
$$

Therefore $E\left(S^{\prime}\right)$ corresponds to the units of the ring of integers of $\mathbb{Q}(\sqrt{d})$ of the form $x+y \frac{M \sqrt{d}}{2}$. It is easy to check that they are of the form proposed above. 
Proposition 2 Suppose that $S_{1}, \ldots, S_{t}$ are matrices which comprise a complete set of (distinct) representatives for $H\left(d M^{2}, L ; \Gamma^{0}(1)\right)$, and $A_{1}, \ldots, A_{r}$ form a complete set of (distinct) representatives for $\mathrm{SL}_{2}(\mathbb{Z}) / \Gamma^{0}(N)$.

1. Assume that either $d \neq-4,-3$ or $M>1$. Then $A_{i}^{t} S_{j} A_{i}$ gives a complete set of distinct representatives for $H\left(d M^{2}, L ; \Gamma^{0}(N)\right)$, i.e.

$\left|H\left(d M^{2}, L ; \Gamma^{0}(N)\right)\right|=t r=\frac{\left|\mathrm{Cl}_{d}(1)\right|}{u(d)} M N \prod_{p \mid M}\left(1-p^{-1}\left(\frac{d}{p}\right)\right) \prod_{p \mid N}(1+1 / p)$,

where $u(d)=1$ if $d \neq-4,-3$, and $u(-3)=3, u(-4)=2$.

2. Assume that $(d, M)=(-4,1)$ and let $N=2^{n_{0}} p_{1}^{n_{1}} \ldots p_{s}^{n_{s}}$ be the prime decomposition of $N$. Then

$$
\left|H\left(-4, L ; \Gamma^{0}(N)\right)\right|=\frac{1}{2}\left(N \prod_{p \mid N}(1+1 / p)+\mathscr{L}_{-4}\right),
$$

where

$$
\mathscr{L}_{-4}= \begin{cases}2^{s} & \text { if } n_{0} \leq 1 \text { and } \forall_{i} p_{i} \equiv 1(\bmod 4) \\ 0 & \text { otherwise }\end{cases}
$$

3. Assume that $(d, M)=(-3,1)$ and let $N=3^{n_{0}} p_{1}^{n_{1}} \ldots p_{s}^{n_{s}}$ be the prime decomposition of $N$. Then

$$
\left|H\left(-3, L ; \Gamma^{0}(N)\right)\right|=\frac{1}{3}\left(N \prod_{p \mid N}(1+1 / p)+2 \mathscr{L}_{-3}\right),
$$

where

$$
\mathscr{L}_{-3}= \begin{cases}2^{s} & \text { if } n_{0} \leq 1 \text { and } \forall_{i} p_{i} \equiv 1(\bmod 6) \\ 0 & \text { otherwise }\end{cases}
$$

Proof Recall that $r=N \prod_{p \mid N}(1+1 / p)$ and

$$
t=\left|\mathrm{Cl}_{d}(M)\right|= \begin{cases}\frac{\left|\mathrm{Cl}_{d}(1)\right|}{u(d)} M \prod_{p \mid M}\left(1-p^{-1}\left(\frac{d}{p}\right)\right) & \text { if } M>1 \\ \left|\mathrm{Cl}_{d}(1)\right| & \text { if } M=1\end{cases}
$$

Each equivalence class in $H\left(d M^{2}, L ; \Gamma^{0}(1)\right)$ (i.e. $j \in\{1, \ldots, t\}$ is fixed) can be written as a union of sets $\left\{{ }^{t} g{ }^{t} A_{i} S_{j} A_{i} g: g \in \Gamma^{0}(N)\right\}$ with $i \in\{1, \ldots, r\}$. The question is whether they are all disjoint. Assume this is not the case for the sets corresponding to $i_{1}$ and $i_{2}$, i.e. assume there exists $g \in \Gamma^{0}(N)$ such that ${ }^{t} A_{i_{1}} S_{j} A_{i_{1}}={ }^{t} g{ }^{t} A_{i_{2}} S_{j} A_{i_{2}} g$. 
Then $S_{j}={ }^{t}\left(A_{i_{2}} g A_{i_{1}}^{-1}\right) S_{j} A_{i_{2}} g A_{i_{1}}^{-1}$, where $A_{i_{2}} g A_{i_{1}}^{-1} \in \mathrm{SL}_{2}(\mathbb{Z})$. Hence, $A_{i_{2}} g A_{i_{1}}^{-1} \in$ $E\left(S_{j}\right)$ and Lemma 3 tells us precisely what these elements may be. The question is whether it does not imply $i_{1}=i_{2}$ and how often this is the case.

1. If $d \neq-4,-3$, or if $M>1$, then $A_{i_{2}} g= \pm A_{i_{1}}$, and so $i_{1}=i_{2}$.

2. If $(d, M)=(-4,1)$, then either $i_{1}=i_{2}$ as above, or $A_{i_{2}} g= \pm \xi_{S_{j}} A_{i_{1}}$.

3. If $(d, M)=(-3,1)$, then we get two additional possibilities: either $A_{i_{2}} g= \pm\left(\frac{1}{2} 1_{2}+\xi_{S_{j}}\right) A_{i_{1}}$ or $A_{i_{2}} g= \pm\left(-\frac{1}{2} 1_{2}+\xi_{S_{j}}\right) A_{i_{1}}$.

Let us check whether the remaining cases may happen when $i_{1} \neq i_{2}$. Without loss of generality we may assume that $L=1$. Observe that both $H\left(-4,1 ; \Gamma^{0}(1)\right)$ and $H\left(-3,1 ; \Gamma^{0}(1)\right)$ contain only one class, namely the one determined by $1_{2}$ and $\left(\begin{array}{cc}1 & 1 / 2 \\ 1 / 2 & 1\end{array}\right)$, respectively. Indeed, each of the elements $\left(\begin{array}{cc}a^{\prime} & b^{\prime} / 2 \\ b^{\prime} / 2 & c^{\prime}\end{array}\right)$ in $H\left(d M^{2}, 1 ; \Gamma^{0}(1)\right)$ can be written uniquely in a reduced form, that is with $\left|b^{\prime}\right| \leq a^{\prime} \leq c^{\prime}$ (e.g. [3], Theorem 2.8). It is easy to see that if $M=1$ and $d=-4,-3$, the only matrix satisfying these conditions is $1_{2}$ and $\left(\begin{array}{cc}1 & 1 / 2 \\ 1 / 2 & 1\end{array}\right)$, respectively. From this observation it also follows that $\left|\mathrm{Cl}_{-4}(1)\right|=\left|\mathrm{Cl}_{-3}(1)\right|=1$.

Choose a set of representatives for $\mathrm{SL}_{2}(\mathbb{Z}) / \Gamma^{0}(N)$ to be

$$
\left\{A_{1}, \ldots, A_{r}\right\}=\left\{\left(\begin{array}{ll}
* u \\
* v
\end{array}\right) \in \mathrm{SL}_{2}(\mathbb{Z}): v \mid N, u \quad(\bmod N / v)\right\}
$$

Let $A_{i_{1}}=\left(\begin{array}{ll}* & u \\ * & v\end{array}\right)$ and $A_{i_{2}}=\left(\begin{array}{c}* u^{\prime} \\ * \\ *\end{array}\right)$.

Assume first that $(d, M)=(-4,1)$. Then $g= \pm A_{i_{2}}^{-1}\left({ }_{-1}{ }^{1}\right) A_{i_{1}}$ is in $\Gamma^{0}(N)$ if and only if $N \mid u u^{\prime}+v v^{\prime}$. Since $\operatorname{gcd}(u, v)=1=\operatorname{gcd}\left(u^{\prime}, v^{\prime}\right)$ and $v, v^{\prime} \mid N$, we must have $v=\operatorname{gcd}\left(u^{\prime}, N\right), v^{\prime}=\operatorname{gcd}(u, N)$ and $\operatorname{gcd}\left(v, v^{\prime}\right)=1$. Put $u=v^{\prime} \underline{u}$ and $u^{\prime}=v \underline{u^{\prime}}$ so that $\frac{N}{v v^{\prime}} \mid \underline{u u^{\prime}}+1$. Note that $\underline{u^{\prime}}$ is determined by $v, v^{\prime}, \underline{u}$. Moreover, under the assumption $\operatorname{gcd}\left(v, v^{\prime}\right)=1, i_{1}=i_{2}$ if and only if $v=v^{\prime}=1$ and $u=u^{\prime}$ satisfies $u^{2}=-1 \bmod N$.

Hence, if we fix $u, v$, then $u^{\prime}, v^{\prime}$ are uniquely determined and thus there are

$$
\frac{N}{2} \prod_{p \mid N}(1+1 / p)+\frac{\mathscr{L}_{-4}}{2}
$$

$\Gamma^{0}(N)$-non-equivalent classes within each class in $H\left(-4, L ; \Gamma^{0}(1)\right)$, where

$$
\mathscr{L}_{-4}:=\#\left\{u \in\{1, \ldots, N\}: u^{2}=-1 \bmod N\right\} .
$$

If $(d, M)=(-3,1)$, then $g= \pm A_{i_{2}}^{-1}\left( \pm \frac{1}{2} I+\left(\begin{array}{cc}1 / 2 & 1 \\ -1 & -1 / 2\end{array}\right)\right) A_{i_{1}}$ is in $\Gamma^{0}(N)$ if and only if

$$
N \mid u u^{\prime}+v v^{\prime}+u^{\prime} v \quad \text { or } \quad N \mid u u^{\prime}+v v^{\prime}+u v^{\prime} .
$$

We will look for the solutions to the first condition, the latter one being symmetric.

From similar reasons as in the previous situation, $u^{\prime}=v \underline{u^{\prime}}$ and $\operatorname{gcd}\left(u^{\prime}, N\right)=1$. Hence, $\operatorname{gcd}\left(v, v^{\prime}\right) \mid u u^{\prime}$, and thus $v$ and $v^{\prime}$ are coprime. Our condition becomes $\frac{N}{v} \mid v^{\prime}+\underline{u^{\prime}}(u+v)$ and implies $v^{\prime}=\operatorname{gcd}(N, u+v)$. Let $t=u+v$ 
and write $t=v^{\prime} \underline{t}$. It is easy to see that $t$ runs through the rests modulo $N / v$ and $\operatorname{gcd}(t, v)=1$. Moreover, $\frac{N}{v v^{\prime}} \mid 1+\underline{u^{\prime} t}$.

Hence, if we fix $v$ and $u, v^{\prime}$ and $u^{\prime}$ are uniquely determined. Similarly, if $N \mid u \tilde{u}+$ $v \tilde{v}+u \tilde{v}$, then $\tilde{u}$ and $\tilde{v}$ are uniquely determined by $u, v$. Moreover, it is easy to check that the conditions ( $\star \star)$ hold at the same time only if $v=v^{\prime}=1$ and $u=u^{\prime}$ satisfies $u^{2}+u+1 \equiv 0(\bmod N)$. Hence the conditions $(\star \star)$ and uniqueness of the solution for each of them imply that unless $v=1$ and $u^{2}+u+1 \equiv 0(\bmod N)$, the matrix $\left(\begin{array}{ll}* & u \\ * & v\end{array}\right)$ is $\Gamma^{0}(N)$-equivalent to exactly two matrices. Therefore, there are

$$
\frac{N}{3} \prod_{p \mid N}(1+1 / p)+2 \frac{\mathscr{L}_{-3}}{3}
$$

$\Gamma^{0}(N)$-non-equivalent classes within each class in $H\left(-3, L ; \Gamma^{0}(1)\right)$, where

$$
\mathscr{L}_{-3}:=\#\left\{u \in\{1, \ldots, N\}: u^{2}+u+1=0 \bmod N\right\} .
$$

In the following lemma we compute the quantities $\mathscr{L}_{-4}$ and $\mathscr{L}_{-3}$, and that finishes the proof.

Lemma 4 1. Let $N=2^{n_{0}} p_{1}^{n_{1}} \ldots p_{s}^{n_{s}}$ be the prime decomposition of $N$. Then

$$
\#\left\{u \in(\mathbb{Z} / N \mathbb{Z})^{\times}: u^{2}=-1 \bmod N\right\}= \begin{cases}2^{s} & \text { if } n_{0} \leq 1 \text { and } \forall_{i} p_{i} \equiv 1(\bmod 4), \\ 0 & \text { otherwise }\end{cases}
$$

2. Let $N=3^{n_{0}} p_{1}^{n_{1}} \ldots p_{s}^{n_{s}}$ be the prime decomposition of $N$. Then

$$
\#\left\{u \in(\mathbb{Z} / N \mathbb{Z})^{\times}: u^{2}+u+1=0 \bmod N\right\}= \begin{cases}2^{s} & \text { if } n_{0} \leq 1 \text { and } \forall_{i} p_{i} \equiv 1(\bmod 6) \\ 0 & \text { otherwise }\end{cases}
$$

Proof This follows from Chinese Remainder Theorem and two basic facts:

- For an odd prime $p,\left(\mathbb{Z} / p^{n} \mathbb{Z}\right)^{\times}$is a cyclic group of order $p^{n-1}(p-1)$.

- $\left(\mathbb{Z} / 2^{n} \mathbb{Z}\right)^{\times}$is cyclic of order 1 and 2 for $n=1$ and 2 , respectively. If $n \geq 3$, then it is the product of two cyclic groups, one of order 2 , the other of order $2^{n-2}$.

1. Depending whether $p \equiv 1(\bmod 4)$ or $p \equiv-1(\bmod 4),-1$ is a square in $\left(\mathbb{Z} / p^{n} \mathbb{Z}\right)^{\times}$or not, respectively. Moreover, because there is only one element of order 2 , there are either 2 or 0 solutions to $u^{2}=-1 \bmod p^{n}$. If $p=2,-1$ is a square in $\left(\mathbb{Z} / 2^{n} \mathbb{Z}\right)^{\times}$only if $n=1$, in which case $-1=1^{2}$.

2. First note that the equation $u^{2}+u+1=0$ has no solution modulo 2 . Furthermore, because the solutions are of the form $(-1+\sqrt{-3}) 2^{-1}$, the equation has one solution modulo 3 and no solutions modulo $3^{n}$ for $n>1$. Now, since $u^{3}-1=$ $(u-1)\left(u^{2}+u+1=0\right)$, we will look for the elements of order 3 in $\left(\mathbb{Z} / p^{n} \mathbb{Z}\right)^{\times}$, where $p \equiv \pm 1(\bmod 6)$.

If $p \equiv-1(\bmod 6)$, then the order of $\left(\mathbb{Z} / p^{n} \mathbb{Z}\right)^{\times}$is not divisible by 3. In the other 
case, there are two elements of order $3, u_{0}$ and $u_{0}^{2}$, say. Both of them are zeros of the polynomial $u^{2}+u+1=0 \bmod p^{n}$.

In Proposition 2 we computed the size of $H\left(d M^{2}, L ; \Gamma^{0}(N)\right)$. On the other hand, we know that ([17], proof of Proposition 5.3) for $M N>1$

$$
\left|H_{1}\left(d M^{2}, L ; \Gamma^{0}(N)\right)\right|=\left|\mathrm{Cl}_{d}(M N)\right|=\frac{\left|\mathrm{Cl}_{d}(1)\right|}{u(d)} M N \prod_{p \mid M N}\left(1-p^{-1}\left(\frac{d}{p}\right)\right),
$$

where $u(d)$ is as in Proposition 2. Combining these results yields the following.

Proposition 3 The map $\tilde{\phi}_{L, M}: \mathrm{Cl}_{d}(M N) \rightarrow H\left(d M^{2}, L ; \Gamma^{0}(N)\right)$ is surjective if and only if $\left(\frac{d M^{2}}{p}\right)=-1$ for all primes $p \mid N$.

Corollary 1 The following conditions are equivalent:

1. $H_{1}\left(d M^{2}, L ; \Gamma^{0}(N)\right)=H\left(d M^{2}, L ; \Gamma^{0}(N)\right)$,

2. $\left(\frac{d M^{2}}{p}\right)=-1$ for all $p \mid N$,

3. $\left|H\left(d M^{2} N^{2}, L ; \Gamma^{0}(1)\right)\right|=\left|H\left(d M^{2}, L ; \Gamma^{0}(N)\right)\right|$.

Proof This follows from the fact that $\mathrm{Cl}_{d}(M N) \cong H\left(d M^{2} N^{2}, L ; \Gamma^{0}(1)\right)$ (Proposition 5.3, [17]) and Propositions 1, 3.

\section{The relation}

Fix $\Phi \in V_{\pi}$ and assume that $\Phi$ is right invariant by the subgroup $I_{N_{1}, N_{2}}$ of $G\left(\mathbb{A}_{f}\right)$ for some $N_{1} \mid N_{2}$.

For any two integers $L, M$, we define the element $H(L, M) \in G(\mathbb{A})$ by

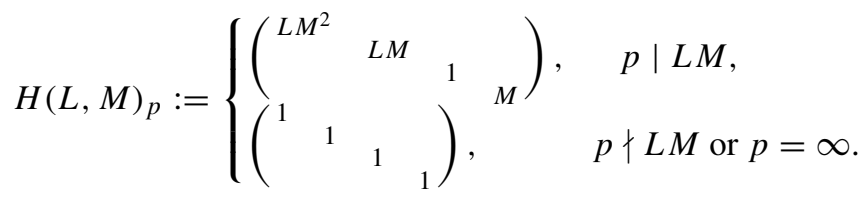

Note that $H(1,1)=1_{4}$ and $\Phi\left(g H(L, M)_{p}\right)=\Phi\left(g h_{p}\left(l_{p}, m_{p}\right)\right)$ for all $p<\infty$, $g \in G(\mathbb{A})$, where $l_{p}=\operatorname{or} d_{p} L$ and $m_{p}=\operatorname{or} d_{p} M$. For the rest of this section we relax the notation and abbreviate $h_{p}(l, m):=h_{p}\left(l_{p}, m_{p}\right)$.

Fix a fundamental discriminant $d<0$ and put $S=S(d), T=T_{S}$. Let $\psi=\prod_{v} \psi_{v}$ be the character of $\mathbb{A}$ such that

- the conductor of $\psi_{p}$ is $\mathbb{Z}_{p}$ for all (finite) primes $p$,

- $\psi_{\infty}(x)=e(x)$, for $x \in \mathbb{R}$,

$-\left.\psi\right|_{\mathbb{Q}}=1$; 
so that $\theta(u(X)):=\psi(\operatorname{tr}(S X))$ is a character of $U(\mathbb{A})$. Let $\Lambda=\prod_{p \leq \infty} \Lambda_{p}$ be a character of $T(\mathbb{A}) / T(\mathbb{Q}) T(\mathbb{R})$ such that $\left.\Lambda\right|_{\mathbb{A}^{\times}}=1$, and let $C(\Lambda)=\prod_{p} p^{c\left(\Lambda_{p}\right)}$ be the smallest integer such that $\left.\Lambda\right|_{T_{C(\Lambda)}}=1$ (cf. Definitions 1, 2). Suppose that $\Phi=\tilde{\Phi} \otimes_{p \nmid N_{2}} \phi_{p}$ is a pure tensor in the space of $\pi$ away from the level. If $\pi$ has a global Bessel model of type $(\Lambda, \theta)$, then for each place $p$ of $\mathbb{Q}, \pi_{p}$ has a local Bessel model of type $\left(\Lambda_{p}, \theta_{p}\right)$ and each $\phi_{p}$ corresponds to a (unique up to multiples) vector $B_{\phi_{p}}$ in the local Bessel model of $\pi_{p}$.

Definition 3 For each $\Phi \in V_{\pi}$ and a character $\Lambda$ such that a global $(\Lambda, \theta)$-Bessel model exists, define

$$
M_{\Phi, \Lambda}:=\prod_{p \nmid N_{2}} p^{c\left(\Lambda_{p}\right)} .
$$

Remark 2 If $\pi_{p}$ is a spherical representation with trivial central character and it is not of type I in notation of [19] (that is, it is of type IIb, IIIb, IVd, Vd or VId), then $\pi_{p}$ admits a local Bessel model if and only if $\Lambda=1$, in which case $c\left(\Lambda_{p}\right)=0$. The only representations that will occur in the proof of main theorem regarding Maass relations are of type IIb, and thus in what follows we could take simply $M_{\Phi, \Lambda}=1$.

The following lemma is the base for our main results.

Lemma 5 Let $L, M, L^{\prime}, M^{\prime}$ be positive integers such that $L^{\prime}\left|L, M_{\Phi, \Lambda}\right| M^{\prime} \mid M$, $\left(L, N_{2}^{\infty}\right)=\left(L^{\prime}, N_{2}^{\infty}\right)$ and $\left(M, N_{2}^{\infty}\right)=\left(M^{\prime}, N_{2}^{\infty}\right)$. If a local $\left(\Lambda_{p}, \theta_{p}\right)$-Bessel model exists at all primes $p \nmid N_{2}$, then the following relation holds:

$$
B_{\Phi}(H(L, M)) \prod_{\substack{p \mid L^{\prime} M^{\prime} \\ p \nmid N_{2}}} B_{\phi_{p}}\left(h_{p}\left(l^{\prime}, m^{\prime}\right)\right)=B_{\Phi}\left(H\left(L^{\prime}, M^{\prime}\right)\right) \prod_{\substack{p \mid L M \\ p \nmid N_{2}}} B_{\phi_{p}}\left(h_{p}(l, m)\right) .(10)
$$

Otherwise, $B_{\Phi}(H(\underline{L}, \underline{M}))=0$ for all integers $\underline{L}, \underline{M}$.

Proof Observe first that if $\pi$ does not have a global Bessel model of type $(\Lambda, \theta)$, then both sides of (10) are zero. We may assume then that the global Bessel model exists.

By uniqueness of local Bessel functionals,

$$
B_{\Phi}(H(L, M))=C \prod_{p \mid N_{2}} B_{\phi_{p}}\left(h_{p}(l, m)\right) \prod_{p \nmid N_{2}} B_{\phi_{p}}\left(h_{p}(l, m)\right)
$$

for any positive integers $L, M$. The constant $C$ can be found if we specialise to $L_{\mathscr{S}}:=$ $\left(L, N_{2}^{\infty}\right)$ and $M_{\Phi, \Lambda} M_{\mathscr{S}}$, where $M_{\mathscr{S}}:=\left(M, N_{2}^{\infty}\right)$ :

$$
B_{\Phi}\left(H\left(L_{\mathscr{S}}, M_{\Phi, \Lambda} M_{\mathscr{S}}\right)\right)=C \prod_{p \mid N_{2}} B_{\phi_{p}}\left(h_{p}(l, m)\right)
$$


(recall that we set the normalisation $B_{\phi_{p}}\left(h_{p}\left(0\right.\right.$ ord $\left.\left.d_{p}\left(M_{\Phi, \Lambda}\right)\right)\right)=1$ for $\left.p \nmid N_{2}\right)$, and thus

$$
B_{\Phi}(H(L, M))=B_{\Phi}\left(H\left(L_{\mathscr{S}}, M_{\Phi, \Lambda} M_{\mathscr{S}}\right)\right) \prod_{\substack{p \mid L M \\ p \nmid N_{2}}} B_{\phi_{p}}\left(h_{p}(l, m)\right)
$$

Using this equation with $L^{\prime}, M^{\prime}$ as in the statement of the lemma, we obtain the relation (10). Note that without the assumption $M_{\Phi, \Lambda} \mid M^{\prime}$ the statement is still true, but we have zeros on both sides of the equality.

From the simple looking relation (10) we obtain a correspondence between the Fourier coefficients (8) that will be crucial for our applications. We start with two auxiliary lemmas.

Lemma 6 Let $A \in \mathrm{GL}_{2}(\mathbb{Q}), \alpha \in \mathbb{Q}^{\times}, \gamma=\left({ }^{A}{ }_{\alpha^{t} A^{-1}}\right)$, let $\gamma_{f}$ be the image of $\gamma$ in $G\left(\mathbb{A}_{f}\right)$, and $\gamma_{\infty}=\gamma \gamma_{f}^{-1}$. Then for any automorphic form $\Phi$ on $G(\mathbb{A})$, any matrix $T \in M_{2}^{\mathrm{sym}}(\mathbb{Q})$ and $g_{\infty} \in G(\mathbb{R})^{+}$we have

$$
\Phi_{T}\left(g_{\infty} \gamma_{f}\right)=\Phi_{\alpha^{-1}{ }^{t} A T A}\left(\gamma_{\infty}^{-1} g_{\infty}\right)
$$

Proof This follows from the fact that $\Phi$ is left $G(\mathbb{Q})$-invariant and the substitution $X=\alpha^{-1} A Y^{t} A$, according to the notation in (8).

Lemma 7 Let $\Phi$ be an automorphic form on $G(\mathbb{A})$ that satisfies Eq. (1), and let $F$ be as in (2). Then for any matrix $T \in M_{2}^{\mathrm{sym}}(\mathbb{Q})$ and $g_{\infty} \in G(\mathbb{R})^{+}$we have

$$
\Phi_{T}\left(g_{\infty}\right)=\mu\left(g_{\infty}\right)^{k} j\left(g_{\infty}, i_{2}\right)^{-k} a(F, T) e\left(\operatorname{tr}\left(T\left(g_{\infty} \cdot i_{2}\right)\right)\right)
$$

Proof By the assumptions and the cocycle relation of the automorphy factor $j$,

$$
\begin{aligned}
\Phi_{T}\left(g_{\infty}\right)= & \int_{M_{2}^{\text {sym }}(\mathbb{Q}) \backslash M_{2}^{\text {sym }}(\mathbb{A})} \psi^{-1}(\operatorname{tr}(T X)) \Phi\left(u(X) g_{\infty}\right) d X \\
= & \int_{M_{2}^{\text {sym }}(\mathbb{Z}) \backslash M_{2}^{\text {sym }}(\mathbb{R})} e(-\operatorname{tr}(T X)) \mu\left(g_{\infty}\right)^{k} j\left(u(X) g_{\infty}, i_{2}\right)^{-k} F\left(u(X) g_{\infty} \cdot i_{2}\right) d X \\
= & \mu\left(g_{\infty}\right)^{k} j\left(g_{\infty}, i_{2}\right)^{-k} \sum_{T^{\prime}} a\left(F, T^{\prime}\right) \\
& \times \int_{M_{2}^{\text {sym }}(\mathbb{Z}) \backslash M_{2}^{\text {sym }}(\mathbb{R})} e(-\operatorname{tr}(T X)) e\left(\operatorname{tr}\left(T^{\prime}\left(g_{\infty} \cdot i_{2}\right)\right)\right) e\left(\operatorname{tr}\left(T^{\prime} X\right)\right) d X \\
= & \mu\left(g_{\infty}\right)^{k} j\left(g_{\infty}, i_{2}\right)^{-k} a(F, T) e\left(\operatorname{tr}\left(T\left(g_{\infty} \cdot i_{2}\right)\right)\right) .
\end{aligned}
$$

Theorem 3 Let $\pi$ be an irreducible cuspidal automorphic representation of $G(\mathbb{A})$ with trivial central character and $\Phi \in V_{\pi}$ an automorphic form in its vector space. Assume 
that $\Phi$ is right $I_{N_{1}, N_{2}}$-invariant for some $N_{1} \mid N_{2}$. Let $S=S(d)$, and $\psi$ as above. Let $\Lambda$ be a character of $T(\mathbb{A}) / T(\mathbb{Q}) T(\mathbb{R})$ such that $\left.\Lambda\right|_{\mathbb{A} \times}=1$. Then for any $L, M, L^{\prime}, M^{\prime}$ satisfying the conditions of Lemma 5 and such that $C(\Lambda) \mid M^{\prime} N_{1}$, we have the following correspondence between the Fourier coefficients of $F$ :

$$
\begin{aligned}
& \frac{\left|\mathrm{Cl}_{d}\left(M^{\prime} N_{1}\right)\right|}{\left|\mathrm{Cl}_{d}\left(M N_{1}\right)\right|} \sum_{T \in H_{1}\left(d M^{2}, L ; \Gamma^{0}\left(N_{1}\right)\right)} \Lambda^{-1}(T) a(F, T) \prod_{\substack{p \mid L^{\prime} M^{\prime} \\
p \nmid N_{2}}} B_{\phi_{p}}\left(h_{p}\left(l^{\prime}, m^{\prime}\right)\right) \\
& =\sum_{T^{\prime} \in H_{1}\left(d M^{\prime 2}, L^{\prime} ; \Gamma^{0}\left(N_{1}\right)\right)} \Lambda^{-1}\left(T^{\prime}\right) a\left(F, T^{\prime}\right) \prod_{\substack{p \mid L M \\
p \nmid N_{2}}} B_{\phi_{p}}\left(h_{p}(l, m)\right) .
\end{aligned}
$$

Proof In view of the relation (10), it suffices to compute the values $B_{\Phi}(H(L, M))$.

Let $\Phi^{L, M}(g):=\Phi(g H(L, M))$ for $g \in G(\mathbb{A})$; because $\Phi$ is right $I_{N_{1}, N_{2}}$-invariant, $\Phi^{L, M}$ is right invariant by

$H_{\infty} I_{N_{1}, N_{2}} H_{\infty}^{-1}=\left\{\prod_{p<\infty}\left(\begin{array}{cccc}* & M N_{1} * & L M^{2} * & L M * \\ * / M & * & L M * & L * \\ * N_{2} / L M^{2} & * N_{2} / L M & * & * / M \\ * N_{2} / L M & * N_{2} / L & M N_{1} * & *\end{array}\right) \in G\left(\mathbb{Q}_{p}\right): * \in \mathbb{Z}_{p}\right\}$

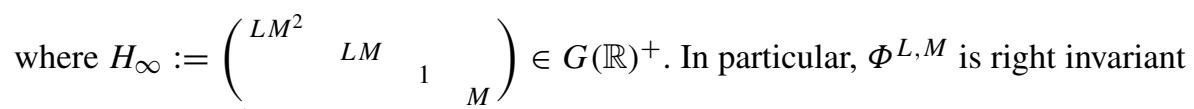
by $T_{M N_{1}}$ and $K_{M N_{1}}^{*}$ (when viewed as subgroups of $G\left(\mathbb{A}_{f}\right)$ via the embedding as in (5)).

Following the notation of Sect. 4, we can write

$$
T(\mathbb{A})=\coprod_{c \in \mathrm{Cl}_{d}\left(M N_{1}\right)} t_{c} T(\mathbb{Q}) T(\mathbb{R}) T_{M N_{1}}
$$

where we choose $t_{c} \in \prod_{p<\infty} T\left(\mathbb{Q}_{p}\right)$, and write $t_{c}=\gamma_{c} m_{c} \kappa_{c}$ with $\gamma_{c} \in \mathrm{GL}_{2}(\mathbb{Q})$, $m_{c} \in \mathrm{GL}_{2}(\mathbb{R})^{+}$and $\kappa_{c} \in K_{M N_{1}}^{*}$.

With this preparation we are ready to compute the values $B_{\Phi}(H(L, M))$.

$$
\begin{aligned}
B_{\Phi}(H(L, M))= & \int_{\mathbb{A}^{\times} T(\mathbb{Q}) \backslash T(\mathbb{A})} \Lambda^{-1}(t) \Phi_{S}^{L, M}(t) d t \\
= & \sum_{c \in \mathrm{Cl}_{d}\left(M N_{1}\right)} \int_{\mathbb{R}^{\times} \backslash T(\mathbb{R})} \Phi_{S}^{L, M}\left(t_{c} t_{\infty}\right) d t_{\infty} \\
& \times \int_{\mathbb{A}_{f}^{\times} T(\mathbb{Q}) \cap T_{M N_{1}} \backslash T_{M N_{1}}} \Lambda^{-1}\left(t_{c} t_{\infty} t_{M N_{1}}\right) d t_{M N_{1}}
\end{aligned}
$$


Observe that if $C(\Lambda) \nmid M N_{1}$, then the inner integral is equal to zero and Eq. (11) holds. Henceforth we assume that $C(\Lambda) \mid M N_{1}$ so that

$$
B_{\Phi}(H(L, M))=\frac{1}{\left|\mathrm{Cl}_{d}\left(M N_{1}\right)\right|} \sum_{c \in \mathrm{Cl}_{d}\left(M N_{1}\right)} \Lambda^{-1}\left(t_{c}\right) \int_{\mathbb{R}^{\times} \backslash T(\mathbb{R})} \Phi_{S}^{L, M}\left(\gamma_{c} m_{c} t_{\infty}\right) d t_{\infty}
$$

Now, applying Lemma 6 twice, we compute the integral:

$$
\begin{aligned}
\int_{\mathbb{R}^{\times} \backslash T(\mathbb{R})} \Phi_{S}^{L, M}\left(\gamma_{c} m_{c} t_{\infty}\right) d t_{\infty} & =\int_{\mathbb{R}^{\times} \backslash T(\mathbb{R})} \underset{S}{L, M}\left(t_{\infty}\left(\gamma_{c}\right)_{f}\right) d t_{\infty} \\
& =\int_{\mathbb{R}^{\times} \backslash T(\mathbb{R})} \Phi_{S_{c}}^{L, M}\left(m_{c} t_{\infty}\right) d t_{\infty} \\
& =\int_{\mathbb{R}^{\times} \backslash T(\mathbb{R})} \underset{\phi_{L, M}(c)}{\Phi_{\infty}}\left(H_{\infty}^{-1} m_{c} t_{\infty}\right) d t_{\infty}
\end{aligned}
$$

where $\phi_{L, M}(c)$ is defined as in (9). Further,

$$
\begin{aligned}
& \int_{\mathbb{R}^{\times} \backslash T(\mathbb{R})} \Phi_{\phi_{L, M}(c)}\left(H_{\infty}^{-1} m_{c} t_{\infty}\right) d t_{\infty} \\
& \stackrel{\text { Lemma }}{=}(L M)^{-k} a\left(F, \phi_{L, M}(c)\right) \int_{\mathbb{R}^{\times} \backslash T(\mathbb{R})} e\left(\operatorname{tr}\left(\phi_{L, M}(c)\left(H_{\infty}^{-1} m_{c} t_{\infty} \cdot i_{2}\right)\right)\right) d t_{\infty} \\
& =(L M)^{-k} a\left(F, \phi_{L, M}(c)\right) \int_{\mathbb{R}^{\times} \backslash T(\mathbb{R})} e\left(\operatorname{tr}\left(S_{c}\left(m_{c} t_{\infty} \cdot i_{2}\right)\right)\right) d t_{\infty} \\
& =(L M)^{-k} a\left(F, \phi_{L, M}(c)\right) \int_{\mathbb{R}^{\times} \backslash T(\mathbb{R})} e\left(\operatorname{tr}\left(S i_{2}\right)\right) d t_{\infty} \\
& =r(L M)^{-k} a\left(F, \phi_{L, M}(c)\right) e^{-2 \pi \operatorname{tr} S},
\end{aligned}
$$

where we set $r:=\int_{\mathbb{R}^{\times} \backslash T(\mathbb{R})} d t_{\infty}$.

Corollary 2 Let $F$ be a Siegel cusp form of degree 2, level $\Gamma_{0}\left(N_{1}, N_{2}\right)$ and weight $k$. Suppose that $F$ is an eigenform of the local Hecke algebra at all primes $p \nmid N_{2}{ }^{6}$ Let $d$ be a fundamental discriminant and let $L, M, L^{\prime}, M^{\prime}$ be positive integers such that $L^{\prime}\left|L, M^{\prime}\right| M,\left(L, N_{2}^{\infty}\right)=\left(L^{\prime}, N_{2}^{\infty}\right)$ and $\left(M, N_{2}^{\infty}\right)=\left(M^{\prime}, N_{2}^{\infty}\right)$. Then for all characters $\Lambda$ of $H_{1}\left(d M^{\prime 2}, L, \Gamma^{0}\left(N_{1}\right)\right) \cong \mathrm{Cl}_{d}\left(M^{\prime} N_{1}\right)$,

$$
\begin{aligned}
& \frac{\left|\mathrm{Cl}_{d}\left(M^{\prime} N_{1}\right)\right|}{\left|\mathrm{Cl}_{d}\left(M N_{1}\right)\right|}\left(\frac{L^{\prime} M^{\prime}}{L M}\right)^{k} \sum_{T \in H_{1}\left(d M^{2}, L ; \Gamma^{0}\left(N_{1}\right)\right)} \Lambda^{-1}(T) a(F, T) \prod_{\substack{p \mid L^{\prime} M^{\prime} \\
p \nmid N_{2}}} B_{\phi_{p}}\left(h_{p}\left(l^{\prime}, m^{\prime}\right)\right) \\
& =\sum_{T^{\prime} \in H_{1}\left(d M^{\prime 2}, L^{\prime} ; \Gamma^{0}\left(N_{1}\right)\right)} \Lambda^{-1}\left(T^{\prime}\right) a\left(F, T^{\prime}\right) \prod_{\substack{p \mid L M \\
p \nmid N_{2}}} B_{\phi_{p}}\left(h_{p}(l, m)\right) .
\end{aligned}
$$

\footnotetext{
${ }^{6}$ It is enough to assume that $F$ is an eigenform of the Hecke operators $T(p)$ and $T\left(p^{2}\right)$ at $p \nmid N_{2}$. For the definition of these Hecke operators see for example [1].
} 
Proof This follows immediately from Theorem 3 and Proposition 3.11 [20], which states that in our setting the following conditions are equivalent:

(i) $F$ is an eigenform of the local Hecke algebra at all primes $p \nmid N_{2}$.

(ii) If $\pi^{\prime}, \pi^{\prime \prime}$ are two irreducible cuspidal representations both of which occur as subrepresentations of the representation $\pi$ associated with $F$, then $\pi_{p}^{\prime} \cong \pi_{p}^{\prime \prime}$ for all primes $p \nmid N_{2}$.

As a result, $F=\sum_{i} F_{i}$, where each $F_{i}$ has the same local data at $p \nmid N_{2}$ and is as in Theorem 3.

\section{Maass relations}

Let $F$ be a cuspidal Siegel modular form invariant under the action of $\Gamma_{0}\left(N_{1}, N_{2}\right)$ that is an eigenform of the local Hecke algebra at primes $p \nmid N_{2}$. Let $\Phi$ be the adelisation of $F$, and $\pi=\otimes_{p} \pi_{p}$ the associated automorphic representation. Suppose that for primes $p \nmid N_{2}, \pi_{p}=\chi_{p} 1_{\mathrm{GL}(2)} \rtimes \chi_{p}^{-1}$ with an unramified character $\chi_{p}$ of $\mathbb{Q}_{p}^{\times}$(a representation of type IIb according to [19]). Note that these are non-tempered, nongeneric representations. The set of $\pi$ obtained in this way is precisely the set of CAP representations attached to the Siegel parabolic subgroup of $G(\mathbb{A})$ (cf. [4]).

Lemma 8 For representation $\pi$ as above, any vector $\tilde{\Phi}=\otimes_{p} \tilde{\phi}_{p}$ in the vector space $V_{\pi}$ of $\pi$ and any non-degenerate matrix $S \in M_{2}^{\mathrm{sym}}(\mathbb{Q})$, we have

$$
\tilde{\Phi}_{S}(t g)=\tilde{\Phi}_{S}(g) \text { for all } g \in G(\mathbb{A}) \text { and } t \in \prod_{p \nmid N_{2}} T_{S}\left(\mathbb{Q}_{p}\right) \prod_{p \mid N_{2}} 1_{4} \text {. }
$$

Proof Let $\tilde{\Phi}=\otimes_{p} \tilde{\phi}_{p}$ be as in the lemma, and let $\mathscr{S}=\left\{p: p \mid N_{2}\right\}$. Without loss of generality we may assume $g=1_{4}$. Let $V^{\mathscr{S}}$ be the subspace of $V_{\pi}$ generated by all vectors of the form $\otimes_{p \in \mathscr{S}} \tilde{\phi}_{p} \otimes_{p \notin \mathscr{S}} \psi_{p}$ with $\psi_{p} \in V_{\pi_{p}}$. The right action of $\otimes_{p \notin \mathscr{S}} G\left(\mathbb{Q}_{p}\right)$ on $V^{\mathscr{S}}$ makes $V^{\mathscr{S}}$ a representation isomorphic to $\otimes_{p \notin \mathscr{S} \pi_{p}}$ (here $\left.\mathbb{Q}_{\infty}:=\mathbb{R}\right)$. Define

$$
\beta: V^{\mathscr{S}} \rightarrow \mathbb{C}, \quad \beta(\Psi):=\Psi_{S}\left(1_{4}\right)=\int_{U(\mathbb{Q}) \backslash U(\mathbb{A})} \Psi(u) \theta_{S}^{-1}(u) d u
$$

Note that $\beta(\pi(t) \Psi)=\Psi_{S}(t)$. We need to show that $\beta(\pi(t) \tilde{\Phi})=\beta(\tilde{\Phi})$ for all $t \in$ $\prod_{p \notin \mathscr{S}} T\left(\mathbb{Q}_{p}\right)$. This is trivial if $\beta \equiv 0$. So assume $\beta \not \equiv 0$. Let

$$
\Phi^{\prime}=\otimes_{p \in \mathscr{S}} \tilde{\phi}_{p} \otimes_{p \notin \mathscr{S}} \phi_{p}^{\prime} \quad \text { be such that } \quad \beta\left(\Phi^{\prime}\right) \neq 0
$$

For each place $p \notin \mathscr{S}$ we get a functional $\beta_{p}$ on $V_{\pi_{p}}$ via

$$
\beta_{p}\left(\psi_{p}\right):=\beta\left(\psi_{p} \otimes_{q \in \mathscr{S}} \tilde{\phi}_{q} \otimes_{q \notin \mathscr{S} \cup\{p\}} \phi_{q}^{\prime}\right)
$$


Then $\beta_{p}\left(\phi_{p}^{\prime}\right) \neq 0$ and thus $\beta_{p}$ is a non-zero functional on $V_{\pi_{p}}$. Clearly, $\beta_{p}$ satisfies

$$
\beta_{p}\left(\pi_{p}(u) \psi_{p}\right)=\theta_{S}(u) \beta_{p}\left(\psi_{p}\right) \quad \text { for all } \quad \psi_{p} \in V_{\pi_{p}} \text { and } u \in U\left(\mathbb{Q}_{p}\right)
$$

By [13, Corollary 4.2], the matrix $S$ satisfies the conditions of [17, Lemma 4.1], and therefore by this lemma

- the space of such functionals $\beta_{p}$ is one-dimensional,

$-\beta_{p}\left(\pi_{p}(t) \psi_{p}\right)=\beta_{p}\left(\psi_{p}\right)$ for all $\psi_{p} \in V_{\pi_{p}}$ and $t \in T\left(\mathbb{Q}_{p}\right)$.

Hence, there exists a constant $C_{\mathscr{S}}$ such that

$$
\beta(\Psi)=C_{\mathscr{S}} \prod_{p \notin \mathscr{S}} \beta_{p}\left(\psi_{p}\right)
$$

whenever $\Psi \in V^{\mathscr{S}}$ corresponds to $\otimes_{p \in \mathscr{S}} \tilde{\phi}_{p} \otimes_{p \notin \mathscr{S}} \psi_{p}$. In this way, $\beta(\pi(t) \tilde{\Phi})=$ $\beta(\tilde{\Phi})$ for all $t \in \prod_{p \notin \mathscr{S}} T\left(\mathbb{Q}_{p}\right)$.

Lemma 9 Let $F, N_{1}$ be as above, $S=S(d)$, and $L, M$ any positive integers. Then for any $c_{1}, c_{2} \in \mathrm{Cl}_{d}\left(M N_{1}\right)$,

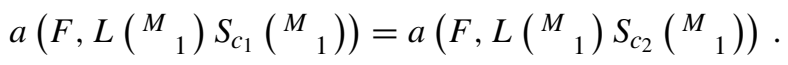

Proof Let $\left\{t_{c}\right\}_{c}$ be a set of representatives of $\mathrm{Cl}_{d}\left(M N_{1}\right)$. We may choose $t_{c}$ so that $t_{c, p}=1_{2}$ for all $p \mid N_{2}$. From the proof of Theorem 3, and using its notation, we get

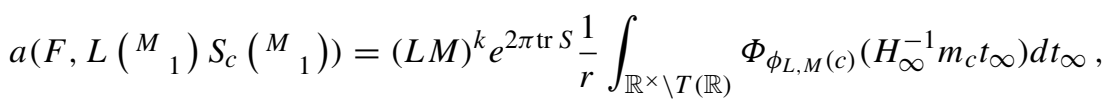

where

$$
\Phi_{\phi_{L, M}(c)}\left(H_{\infty}^{-1} m_{c} t_{\infty}\right)=\Phi_{S}\left(t_{c} t_{\infty} H(L, M)\right) \stackrel{\text { Lemma }}{=} \Phi_{S}\left(t_{\infty} H(L, M)\right)
$$

does not depend on $c$.

Hence, it makes sense to write $a\left(F ; d M^{2}, L\right)$ for any Fourier coefficient of $F$ that

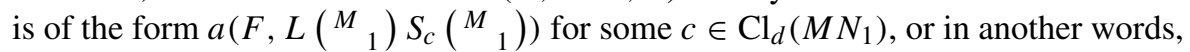
for $a(F, T)$ with $T \in H_{1}\left(d M^{2}, L ; \Gamma^{0}\left(N_{1}\right)\right)$.

The following theorem generalises [17, Theorem 5.1] to cuspidal Siegel modular forms of level $\Gamma_{0}\left(N_{1}, N_{2}\right)$ with $N_{2}>1$.

Theorem 4 Let $F$ be as above. For any fundamental discriminant $d$ and any positive integers L, M, Fourier coefficients of $F$ satisfy the following Maass relations:

$$
a\left(F ; d M^{2}, L\right)=\sum_{\substack{r \mid L \\ \operatorname{gcd}\left(r, N_{2}\right)=1}} r^{k-1} a\left(F ; d\left(\frac{L M}{r L \mathscr{S}}\right)^{2}, L \mathscr{S}\right),
$$


where $L_{\mathscr{S}}=\left(L, N_{2}^{\infty}\right)$.

Hence, if

$$
\left(\begin{array}{cc}
a & b / 2 \\
b / 2 & c
\end{array}\right)=L\left(\begin{array}{cc}
M & \\
& 1
\end{array}\right) S_{\tilde{c}}\left(\begin{array}{cc}
M & \\
& 1
\end{array}\right)
$$

for some $\tilde{c} \in \mathrm{Cl}_{d}\left(M N_{1}\right)$, then

$$
a\left(F,\left(\begin{array}{cc}
a & b / 2 \\
b / 2 & c
\end{array}\right)\right)=\sum_{\substack{r \operatorname{gcd}(a, b, c) \\
\operatorname{gcd}\left(r, N_{2}\right)=1}} r^{k-1} a\left(F, L_{\mathscr{S}}\left(\begin{array}{cc}
\frac{a c}{\left(r L_{\mathscr{C}}\right)^{2}} & \frac{b}{2 r L_{\mathscr{S}}} \\
\frac{2 r L_{\mathscr{S}}}{2 r} & 1
\end{array}\right)\right),
$$

where $L_{\mathscr{S}}=\left(\operatorname{gcd}(a, b, c), N_{2}^{\infty}\right)$.

Proof Recall Corollary 2. If we take $\Lambda$ to be a trivial character ${ }^{7}$ in the formula (12), and $M^{\prime}=\left(M, N_{2}^{\infty}\right), L^{\prime}=\left(L, N_{2}^{\infty}\right)$, then

$$
a\left(F ; d M^{2}, L\right)=\left(\frac{L M}{L^{\prime} M^{\prime}}\right)^{k} a\left(F ; d M^{\prime 2}, L^{\prime}\right) \prod_{\substack{p \mid L M \\ p \nmid N_{2}}} B_{\phi_{p}}\left(h_{p}\left(l_{p}, m_{p}\right)\right),
$$

where $l_{p}=\operatorname{ord} d_{p} L, m_{p}=\operatorname{ord} d_{p} M$. Similarly, for any divisor $r$ of $L$,

$a\left(F ; d\left(\frac{L M}{r L^{\prime}}\right)^{2}, L^{\prime}\right)=\left(\frac{L M}{r L^{\prime} M^{\prime}}\right)^{k} a\left(F ; d M^{\prime 2}, L^{\prime}\right) \prod_{\substack{p \mid L M / r \\ p \nmid N_{2}}} B_{\phi_{p}}\left(h_{p}\left(0, l_{p}+m_{p}-r_{p}\right)\right)$.

Note that the last product can actually be taken over all primes $p \mid L M$ that do not divide $N_{2}$. Indeed, in the product over $p \mid L M / r, p \nmid N_{2}$ we miss only those places $p$ for which both $r_{p}=l_{p}$ and $m_{p}=0$. But in this case $B_{\phi_{p}}\left(h_{p}\left(0, l_{p}+m_{p}-r_{p}\right)\right)=$ $B_{\phi_{p}}\left(h_{p}(0,0)\right)=1$ by Theorem 2 .

Moreover, it is known [17, Theorem 2.1] that the spherical vectors of the representations of type IIb satisfy the equation

$$
B_{p}(h(l, m))=\sum_{i=0}^{l} p^{-i} B_{p}(h(0, l+m-i))
$$

for all $l, m \geq 0$. Hence, Eq. (13) holds if and only if

$$
\prod_{\substack{p \mid L M \\ p \nmid N_{2}}} \sum_{i=0}^{l_{p}} p^{-i} B_{\phi_{p}}\left(h_{p}\left(0, l_{p}+m_{p}-i\right)\right)=\sum_{\substack{r \mid L \\ \operatorname{gcd}\left(r, N_{2}\right)=1}} \frac{1}{r} \prod_{\substack{p \mid L M \\ p \nmid N_{2}}} B_{\phi_{p}}\left(h_{p}\left(0, l_{p}+m_{p}-r_{p}\right)\right),
$$

\footnotetext{
$\overline{7 \text { Since at } p \nmid N_{2}, \pi_{p}=\chi_{p} 1_{\mathrm{GL}}(2)} \rtimes \chi_{p}^{-1}$ is a representation of type IIb, a local $\left(\Lambda_{p}, \theta_{p}\right)$-Bessel model exists if and only if $\Lambda_{p}=1$. See also Remark 2 .
} 
which is true.

It is unfortunate that our method gives us an access only to the coefficients of the form (14), which are not yet fully characterised. Nevertheless, their partial study in section 4 with subsequent Corollary 1 already leads to a satisfactory result which generalises the full level case:

Corollary 3 Let $F, N_{1}, N_{2}$ be as above. Then for any matrix $T=L\left(\begin{array}{cc}a & b / 2 \\ b / 2 & c\end{array}\right)$ for which $L \mid N_{2}^{\infty}, \operatorname{gcd}\left(a, b, c, N_{2}\right)=1$ and $\left(\frac{b^{2}-4 a c}{p}\right)=-1$ for every $p \mid N_{1}$, we have

$$
a\left(F, L\left(\begin{array}{cc}
a & b / 2 \\
b / 2 & c
\end{array}\right)\right)=\sum_{r \mid \operatorname{gcd}(a, b, c)} r^{k-1} a\left(F, L\left(\begin{array}{cc}
\frac{a c}{r^{2}} & \frac{b}{2 r} \\
\frac{b}{2 r} & 1
\end{array}\right)\right)
$$

Acknowledgements The work presented in this paper was carried out at the University of Bristol and represents a part of the $\mathrm{PhD}$ thesis of the author. She would like to thank her supervisor Abhishek Saha for guidance, support and patience in explaining various subtleties.

Open Access This article is licensed under a Creative Commons Attribution 4.0 International License, which permits use, sharing, adaptation, distribution and reproduction in any medium or format, as long as you give appropriate credit to the original author(s) and the source, provide a link to the Creative Commons licence, and indicate if changes were made. The images or other third party material in this article are included in the article's Creative Commons licence, unless indicated otherwise in a credit line to the material. If material is not included in the article's Creative Commons licence and your intended use is not permitted by statutory regulation or exceeds the permitted use, you will need to obtain permission directly from the copyright holder. To view a copy of this licence, visit http://creativecommons.org/licenses/by/4.0/.

\section{References}

1. Andrianov, A. N., Zhuravlëv, V. G.: Modular forms and Hecke operators. Translations of Mathematical Monographs, vol. 145. Translated from the 1990 Russian original by Neal Koblitz. AMS, Providence (1995)

2. Chen, S.Y.: Pullback formulae for nearly holomorphic Saito-Kurokawa lifts. Manuscr. Math. (2019). https://doi.org/10.1007/s00229-019-01111-2

3. Cox, D.A.: Primes of the Form $x^{2}+n y^{2}$. Pure and Applied Mathematics, 2nd edn. Wiley, Hoboken (2013)

4. Dickson, M., Pitale, A., Saha, A., Schmidt, R.: Explicit refinements of Böcherer's conjecture for Siegel modular forms of square-free level. J. Math. Soc. Jpn. arXiv:1512.07204 (2019)

5. Eichler, M., Zagier, D.: The theory of Jacobi forms. Progress in Mathematics, vol. 55. Birkhäuser Boston Inc., Boston (1985)

6. Furusawa, M.: On $L$-functions for GSp(4) $\times \mathrm{GL}(2)$ and their special values. J. Reine Angew. Math. 438, 187-218 (1993)

7. Ibukiyama, T.: Saito-Kurokawa liftings of level $N$ and practical constructions of Jacobi forms. Kyoto J. Math. 52(1), 141-178 (2012)

8. Ichino, A.: Pullbacks of Saito-Kurokawa lifts. Invent. math. 162(3), 551-647 (2005)

9. Kowalski, E., Saha, A., Tsimerman, J.: Local spectral equidistribution for Siegel modular forms and applications. Compos. Math. 148(2), 335-384 (2012)

10. Maass, H.: Über eine Spezialschar von Modulformen zweiten Grades. Invent. Math. 52(1), 95-104 (1979)

11. Novodvorski, M.E., Piatetski-Shapiro, I.I.: Generalized Bessel models for the symplectic group of rank 2. Math. USSR-Sbomik 90, 246-256 (1973)

12. Piatetski-Shapiro, I.I.: On the Saito-Kurokawa lifting. Invent. math. 71, 309-338 (1983) 
13. Pitale, A., Schmidt, R.: Bessel models for lowest weight representations of GSp(4, R ). Int. Math. Res. Not. 7, 1159-1212 (2009)

14. Pitale, A., Schmidt, R.: Integral Representation for $L$-functions for GSp $4 \times \mathrm{GL}_{2}$. J. Number Theory 129(10), 1272-1324 (2009)

15. Pitale, A., Schmidt, R.: Ramanujan-type results for Siegel cusp forms of degree 2. J. Ramanujan Math. Soc. 24(1), 87-111 (2009)

16. Pitale, A., Schmidt, R.: Bessel models for GSp(4): Siegel vectors of square-free level. J. Number Theory 136, 134-164 (2014)

17. Pitale, A., Saha, A., Schmidt, R.: Local and global Maass relations. Math. Z. 287(1-2), 655-677 (2017). https://doi.org/10.1007/s00209-016-1840-5

18. Prasad, D., Takloo-Bighash, R.: Bessel models for GSp(4). J. Reine Angew. Math. 655, 189-243 (2011)

19. Roberts, B., Schmidt, R.: Some results on Bessel functionals for GSp(4). Doc. Math. 21, 467-553 (2016)

20. Saha, A.: On ratios of Petersson norms for Yoshida lifts. Forum Math. 27(4), 2361-2412 (2015)

21. Schmidt, R.: The Saito-Kurokawa lifting and functoriality. Am. J. Math. 127(1), 209-240 (2005)

22. Schmidt, R.: On classical Saito-Kurokawa liftings. J. Reine Angew. Math. 604, 211-236 (2007)

23. Sugano, T.: On holomorphic cusp forms on quaternion unitary groups of degree 2. Fac. Sci. Univ. Tokyo Sect. IA Math. 31(3), 521-568 (1985)

Publisher's Note Springer Nature remains neutral with regard to jurisdictional claims in published maps and institutional affiliations. 